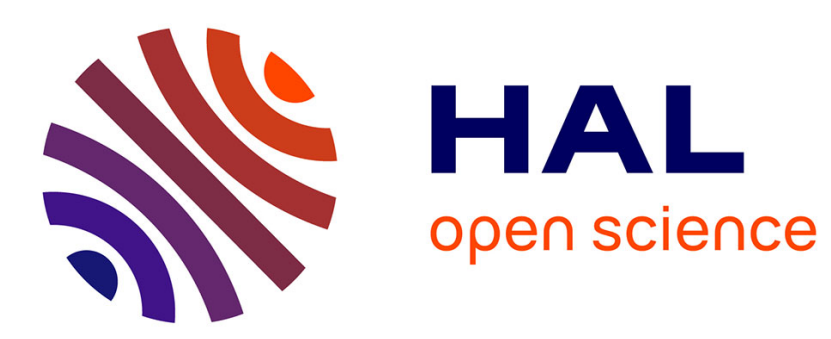

\title{
Risk-Based Clustering for Near Misses Identification in Integrated Deterministic and Probabilistic Safety Analysis
}

Francesco Di Maio, Matteo Vagnoli, Enrico Zio

\section{To cite this version:}

Francesco Di Maio, Matteo Vagnoli, Enrico Zio. Risk-Based Clustering for Near Misses Identification in Integrated Deterministic and Probabilistic Safety Analysis. 2016. hal-01265879

\section{HAL Id: hal-01265879 \\ https://hal.science/hal-01265879}

Preprint submitted on 1 Feb 2016

HAL is a multi-disciplinary open access archive for the deposit and dissemination of scientific research documents, whether they are published or not. The documents may come from teaching and research institutions in France or abroad, or from public or private research centers.
L'archive ouverte pluridisciplinaire HAL, est destinée au dépôt et à la diffusion de documents scientifiques de niveau recherche, publiés ou non, émanant des établissements d'enseignement et de recherche français ou étrangers, des laboratoires publics ou privés. 


\title{
RISK-BASED CLUSTERING FOR NEAR MISSES IDENTIFICATION IN INTEGRATED DETERMINISTIC AND PROBABILISTIC SAFETY \\ ANALYSIS
}

Francesco Di Maio ${ }^{1}$, Matteo Vagnoli ${ }^{1}$, Enrico Zio ${ }^{1,2}$

${ }^{1}$ Energy Department, Politecnico di Milano

Via Ponzio 34/3, 20133 Milano, Italy

francesco.dimaio@polimi.it

${ }^{2}$ Chair on System Science and Energetic Challenge

European Foundation for New Energy - Electricite de France

Ecole Centrale, Paris, and Supelec, Paris, France

\begin{abstract}
In Integrated Deterministic and Probabilistic Safety Analysis (IDPSA), safe scenarios and Prime Implicants (PIs), i.e., minimum combinations of failure events that are capable of leading the system into a fault state are generated by simulation. Post-processing is needed to extract relevant information from these scenarios. In this paper, we propose a novel post-processing method which resorts to a risk-based clustering method for identifying Near Misses among the safe scenarios, i.e., combinations of failure events that lead the system to a quasi-fault state, a condition close to accident. This is important because the possibility of recovering these combinations of failures within a tolerable grace time allows avoiding deviations to accident and, thus, reducing the downtime (and the risk) of the system. The early identification of Near Misses can, then, be useful for online integrated risk monitoring, for rapidly detecting the incipient problems and setting up the recovery strategy of the occurred failures. The post-processing risk-significant features for the clustering are extracted from: i) the probability of a scenario to develop into an accidental scenario, ii) the severity of the consequences that the developing scenario would cause to the system, iii) the combination of i) and ii) into the overall risk of the developing scenario. The optimal selection of the extracted features is done by a wrapper approach, whereby a Modified Binary Differential Evolution (MBDE) embeds a K-means clustering algorithm. The characteristics of the Near Misses scenarios are identified solving a multi-objective optimization problem, using the Hamming distance as a measure of similarity. The feasibility of the analysis is shown with respect to fault scenarios in a dynamic Steam Generator (SG) of a Nuclear Power Plant (NPP).
\end{abstract}


NOMENCLATURE

\section{Abbreviations}

CET Continuous Event Tree

$\mathrm{CH} \quad$ Calinski-Harabasz index

DBA Design Basis Accident

DET Dynamic Event Tree

DSA Deterministic Safety Analysis

ET Event Tree

FT

Fault Tree

IDPSA Integrated Deterministic and Probabilistic Safety Analysis

IE Initiating Event

MBDE Modified Binary Differential Evolution

MCS Minimal Cuts Set

MOP Multi-Objective optimization Problem

MVL Multiple-Valued Logic

NPP Nuclear Power Plant

PIs Prime Implicants

PSA Probabilistic Safety Analysis

SCP Set Covering Problem

SG Steam Generator

TH Thermal-Hydraulics

UTSG U-Tube Steam Generator 


\section{Symbols}

$p$

c

$r$

$t$

$p(t)$

$c(t)$

Probability that the developing scenario is an accidental scenario

Consequence that the developing scenario can cause to the system

Overall risk of the developing scenario

Time instant

Probability that at time $t$ the scenario can lead the system into an accidental scenario

Consequence that at time $t$ the developing scenario is predicted to cause to the system

$r(t) \quad$ Overall risk of the developing scenario at time $t$

$Q_{e} \quad$ Flow-rate of fresh feed-water entering the steam generator

$P_{o} \quad$ Operating power

$P_{n} \quad$ Nominal power

$Q_{v} \quad$ Flow-rate of dry steam exiting the steam generator.

$N_{r l} \quad$ Narrow Range steam generator water Level

$W_{r l} \quad$ Wide Range steam generator water Level

$T_{n} \quad$ Time constant for the $N_{r l}$ dynamics

$Q_{e f} \quad$ Flow-rate of incoming water in steam generator tube bundle region.

$T_{h} \quad$ Time constant for the water mass transportation dynamics

$\tau$

Time constant for the feed-water valve dynamics

$Q_{G V}$

Flow-rate of steam-water mixture exiting the steam generator tube bundle region

$T_{g}$

Time constant for the dynamics relating $Q_{V}$ to $Q_{G V}$

$F_{g}$

Constant in the non-minimum phase term of the dynamics relating $Q_{V}$ to $Q_{G V}$ 


\begin{tabular}{|c|c|}
\hline$T_{\text {int }}$ & Time constant for the $W_{r l}$ dynamics \\
\hline$x$ & System state \\
\hline$\dot{x}$ & Derivative of system state \\
\hline$N_{\text {ref }}$ & Narrow Range steam generator water Level at a reference position \\
\hline$N_{\text {high }}$ & Automatic reactor trip threshold \\
\hline$N_{\text {low }}$ & Turbine trip threshold \\
\hline$N_{h l}$ & First pre-alarm automatic reactor trip threshold \\
\hline$N_{l l}$ & First pre-alarm turbine trip threshold \\
\hline$N_{v h}$ & Second pre-alarm automatic reactor trip threshold \\
\hline$N_{v l}$ & First pre-alarm turbine trip threshold \\
\hline$Q_{\text {pid }}$ & Water flow rate provided by PID controller \\
\hline$Q_{s f}$ & Water flow rate removed by safety valve \\
\hline$T_{\text {miss }}$ & Mission time \\
\hline$t_{m v l}$ & Time steps in MVL discretization \\
\hline$\varphi$ & Cumulative probability function of the Gaussian distribution \\
\hline$\mu$ & Mean value of the Gaussian distribution \\
\hline$\sigma$ & Standard deviation of the Gaussian distribution \\
\hline A & Intensity coefficient \\
\hline K & Number of clusters \\
\hline$n$ & Index of the profile of $p, c$ and $r$ \\
\hline$\mu_{n}$ & Mean value of the $n-t h$ profile \\
\hline $\max$ & Peak value of the $n-t h$ profile \\
\hline
\end{tabular}




\begin{tabular}{|c|c|}
\hline RMS & Root mean square of the $n-t h$ profile \\
\hline$S K$ & Skewness of the $n-t h$ profile \\
\hline$K U$ & Kurtosis of the $n-t h$ profile \\
\hline$N$ & Number of scenarios belogging to the training set \\
\hline$F$ & Dimension of the set of features \\
\hline$T_{\text {set }}$ & Number of scenarios belogging to the test set \\
\hline$S S_{b}$ & Overall between-cluster variance \\
\hline$S S_{w}$ & Overall within-cluster variance \\
\hline$n_{k}$ & Number of scenarios assigned to the $k$-th cluster \\
\hline$x_{c}$ & Generic scenario \\
\hline$m_{k}$ & Centroid of the $k$-th cluster \\
\hline$\mu_{\text {risk }}$ & Mean risk of the clustered scenarios \\
\hline \multirow[t]{2}{*}{$t_{\text {risk }}$} & Time elapsed from the instant at which $r$ starts to deviate from zero of the \\
\hline & clustered scenarios \\
\hline$f$ & Fitness function of the MOP \\
\hline$f_{1}$ & First objective function of the MOP \\
\hline$f_{2}$ & Second objective function of the MOP \\
\hline$\overline{\boldsymbol{x}}$ & Sequence vector belonging to the Pareto set of the MOP \\
\hline
\end{tabular}

\section{INTRODUCTION}

Integrated Deterministic and Probabilistic Safety Analysis (IDPSA) attempts at overcoming some limitations of Deterministic Safety Analysis (DSA) and Probabilistic Safety Analysis (PSA). The former, is solidly founded on by the multi-barrier and defense-in-depth concepts, and aims at verifying the capability of a Nuclear Power Plant (NPP) to withstand a set of postulated Design Basis 
Accidents (DBA) [Kang et al, 2013; Zio et al., 2012a]. To account for the uncertainties in the model representation of the actual plant behavior, conservatism is introduced in the calculations by ThermalHydraulics (TH) codes under DBA conditions [Zio et al., 2010]. The latter aims at considering a wider set of possible accidental scenarios, and includes the quantification of accident probabilities [Aldemir, 2013; Keller et al., 2005].

Both DSA and PSA are scenario-based analyses, where scenario selection and definition are done by expert judgment. State-of-the-art of DSA and PSA approaches can provide relevant and important insights on what is already known to be an "issue", but they are not capable of revealing what, and to what extent, is not known (i.e., scenarios which are not expert-selected in the DSA and PSA input), with the risk of neglecting or underestimating potentially dangerous scenarios [Kudinov et al., 2011]. This is due to the difficulties of the static structure of the classic DSA and PSA approaches in treating dynamic variations that usually occur during the operational time of a process [Khakzad et al., 2012] due to (i) stochastic disturbances (e.g., equipment failures), (ii) deterministic plant responses (i.e., transients), (iii) controls and (iv) operator actions [Marseguerra et al., 1996; Kirschenbaum et al., 2009; Kudinov et al., 2011]. Indeed, the order and timing of the events occurring along a scenario, and the values of the process variables at the time of event occurrence are critical in determining the evolution of the scenario itself [Aldemir et al., 2008].

The development and application of IDPSA in practice must meet the challenge of computational complexity, both in model construction and implementation, and in post-processing for the retrieval of the relevant information from the scenario outcomes. The number of dynamic scenario branches generated in IDPSA increases in power law with the number of occurring events and, thus, is much larger than in classical PSA based on Event Trees (ET) and Fault Trees (FT). The a posteriori information retrieval (post-processing), then, becomes quite burdensome and difficult [Labeau et al., 2000; Zio, 2014]. Continuous Event Trees (CETs) [Devooght et al., 1992; Kopustinskas et al., 2005] and Dynamic Event Trees (DETs) [Hofer et al., 2004; Hakobyan et al., 2008] provide realistic frameworks for IDPSA. However, their application is limited by their computationally intensive nature, by the need of tailoring the algorithms to the system under consideration and by the need of processing a massive amount of data for any single initiating event considered [Di Maio et al., 2014a]. Post-processing, in general, consists in classifying the generated dynamic scenarios into safe scenarios and Prime Implicants (PIs), i.e., sequences of events that represent minimal combinations of accident failures necessary for system failure and cannot be covered by more general implicants [Quine, 1952]. Among the safe scenarios, Near Misses are important scenarios to be identified, because they are those sequences of events that reach values of the safety parameters close to, but not exceeding, the corresponding acceptable thresholds [Zio et al., 2009]. They can, thus, be relevant 
contributors to the "hidden" risk of the system, and should not be neglected, as a small deviation may transform them into accidental scenarios.

In literature, several authors introduce the concept of Near Misses as accident precursors [Bier et al., 1995; Johnson et al., 1996]. We here consider Near Misses as sequences of events that incidentally keep the system in a safe state, but endangered and insecure. For the purpose of the analysis, they are here defined as sequences of events similar to those leading the system into fault conditions, except for one characteristic which is missing or is slightly different (e.g., sequence time lag, different failure magnitude, different involved component in an event) [Saleh et al., 2013].

The post-processing analysis entails a "Forward" classification of the dynamic scenarios into classes, i.e., safe, PIs and Near Misses, and a "Backward" identification of the similarities of the features of the scenarios (i.e., stochastic event occurrence and deterministic process variables values), which characterize the groups of Near Misses among the whole set of safe scenarios.

For the "Forward" classification of the Near Misses sequences, we look at two factors of risk: the probability of occurrence of an undesired event and the severity of the consequence caused by the event [Zadakbar et al., 2013]. Thus, we describe the sequences of events by: i) the probability ( $p$ ) that the developing scenario is an accidental scenario, ii) the consequence $(c)$ that the developing scenario can cause to the system, and iii) the overall risk $(r)$ of the developing scenario, that we compute synthetically as $r=p \times c$ (expected consequence).

The optimal features for discerning the Near Misses from the safe scenarios are extracted from the profiles of $p, c$, and $r$ of the accidental scenarios and selected by a wrapper algorithm, which takes into account six statistical indicators of $p, c$, and $r$, and, through a Modified Binary Differential Evolution (MBDE) optimization algorithm, selects the best features, which are fed to a K-means clustering algorithm, which is a simple and well-known clustering algorithm (other classical clustering algorithms, such as mean-shift [Fukunaga et al., 1975; Mandelli et al., 2010] or fuzzy Cmeans [Bezdek, 1981; Zio et al., 2009]).

The outcomes of this "Forward" classification is, then, interpreted by a "Backward" identification of the similarities of the features of the Near Misses scenarios: the acquired knowledge can be exploited in an online integrated risk monitoring system that can rapidly detect the problem and set up a repair strategy of the occurred failures before that the system reaches a fault state.

The proposed approach is illustrated with reference to scenarios occurring in the Steam Generator (SG) of a NPP [Aubry et al., 2012]. We use Multiple-Valued Logic (MVL) theory for modeling the behavior of the system, where timing and sequences of component failure events are determining the system behavior [Aldemir, 2013]. By using MVL, we increase the limited description capability of binary variables in modeling the different component operational states (for example, a valve that can 
be closed, partially closed or fully open, or can fail at different times) and, therefore, perform an IDPSA post-processing analysis on the whole set of simulated accidental scenarios [Di Maio et al., 2014a].

The paper is organized as follows. In Section 2, the SG model used to generate the scenarios for the reliability analysis is presented [Aubry et al., 2012], along with multistate representation of the system dynamics. In Section 3, the PIs are identified and the risk-based "Forward" and "Backward" Near Misses identification method is introduced with reference to the case study considered. In Section 4, conclusions and remarks are drawn.

\section{CASE STUDY}

\subsection{The U-Tube Steam Generator (UTSG) model}

The U-Tube Steam Generator (UTSG) under consideration is sketched in Fig. 1. The improper control of the water level, whose difficulties arises from non-minimum phase plant characteristics, i.e., plant strong inverse response behavior, particularly at low operating power, due to the so-called "swell and shrink" effects [Kothare et al., 2000], is a major cause of NPP unavailability [Kothare et al., 2000; Habibiyan et al., 2004; Marseguerra et al., 2007].

The reactor coolant enters the UTSG at the bottom, moves upward and then downward in the inverted U-tubes, transferring heat to the secondary fluid before exiting at the bottom. The secondary fluid, the feedwater $\left(Q_{e}\right)$, enters the UTSG at the top of the downcomer, through the space between the tube bundle wrapper and the SG shell. The value of $Q_{e}$ is regulated by a system of valves: a low flow rate valve, used when the operating power $\left(P_{o}\right)$ is smaller than $15 \%$ of nominal power $\left(P_{n}\right)$, and a high flow rate valve when $P_{o}>0.15 P_{n}$ [Aubry et al., 2012]. In the secondary side of the tube bundle, water heats up, reaches saturation, starts boiling and turns into a two-phase mixture. The two-phase fluid moves up through the separator/riser section, where steam is separated from liquid water, and through the dryers, which ensure that the exiting steam $\left(Q_{v}\right)$ is essentially dry. The separated water is recirculated back to the downcomer. The balance between the exiting $Q_{v}$ and the incoming $Q_{e}$ governs the change in the water level in the SG. Because of the two-phase nature, two types of water level measurements are considered, as shown in Fig. 1, each reflecting a different level concept: the Narrow Range Level $\left(N_{r l}\right)$ is calculated by pressure difference between two points close to the water level and indicates the mixture level, whereas, the Wide Range Level $\left(W_{r l}\right)$ is calculated by pressure 
difference between the two extremities of the SG (steam dome and bottom of the downcomer) and indicates the collapsed liquid level that is related with the mass of water in the SG.

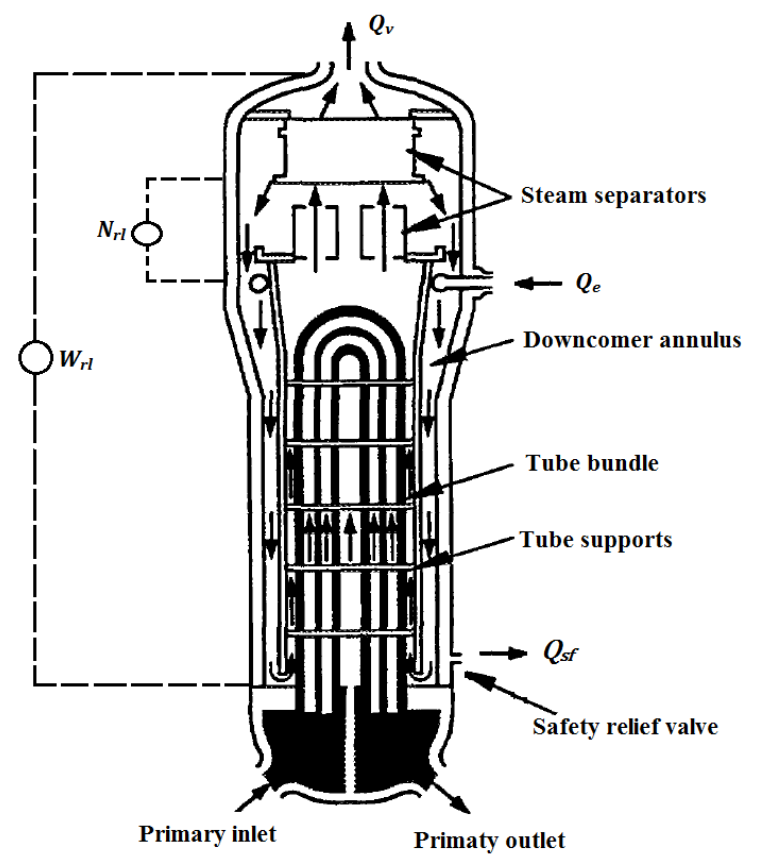

Fig. 1. Schematic of the UTSG [IAEA-TECDOC-981, 1997]

"Swell and shrink" phenomena are also modeled to reproduce the dynamic behavior of the SG: when $Q_{v}$ increases, the steam pressure in the steam dome decreases and the two-phase fluid in the tube bundle expands causing $N_{r l}$ to initially swell (i.e., rise), instead of decreasing as would have been expected by the mass balance; contrarily, if $Q_{v}$ decreases or $Q_{e}$ increases, a shrink effect occurs. A similar model has been presented in [Aubry et al., 2012].

The $N_{r l}$ is governed by $Q_{e}$ and $Q_{v}$ across the tube bundle region of the SG as shown by the following transfer function:

$$
N_{r l}(\mathrm{~s})=\frac{1}{T_{n} s}\left(Q_{e f}(s)-Q_{G V}(s)\right)
$$

where $Q_{e f}$ is the flow-rate of the incoming water in the tube bundle, (Eq. (2)), $Q_{G V}$ is the equivalent steam-water mixture flow-rate exiting the tube bundle region, (Eq. (3)), $T_{n}$ is a time constant that accounts for the $N_{r l}$ dynamics.

The incoming water flow-rate $Q_{e f}$ is proportional to $Q_{e}$ : 


$$
Q_{e f}(\mathrm{~s})=\frac{1}{\left(1+T_{h} s\right)(1+\tau s)} Q_{e}(s)
$$

where the lag $1 /(1+\tau s)$ accounts for the feed-water valve dynamics and $1 /\left(1+T_{h} s\right)$ accounts for the water mass transportation dynamics: their values are reported in Table 1.

The exiting steam-water mass $Q_{G V}$ is proportional to $Q_{v}$ :

$$
Q_{G V}(\mathrm{~s})=\frac{\left(1-F_{g} T_{g} s\right)}{\left(1+T_{g} s\right)} Q_{v}(s)
$$

where the first order lag $1 /\left(1+T_{g} s\right)$ accounts for the elapsed time from the turbine steam demand and the increase of $Q_{G V}$, and the non-minimum phase term $\left(1-F_{g} T_{g} s\right)$ accounts for the two-phase swell and shrink effects.

Combining Eqs. (1), (2), and (3), $N_{r l}$ is equal to:

$$
N_{r l}(\mathrm{~s})=\frac{1}{T_{n} s}\left(\frac{Q_{e}(s)}{\left(1+T_{h} s\right)(1+\tau s)}-\frac{\left(1-F_{g} T_{g} s\right)}{\left(1+T_{g} s\right)} Q_{v}(s)\right)
$$

and $W_{r l}$, i.e., the overall water mass in the steam generator, is:

$$
W_{r l}(\mathrm{~s})=\frac{1}{T_{\text {int }} s}\left(Q_{e}(s)-Q_{v}(s)\right)
$$

where $T_{\text {int }}$ is a time constant that accounts for the $W_{r l}$ dynamics.

We assume $y_{1}=N_{r l}$ and $y_{2}=W_{r l}$, and $u=Q_{e}$ and $d=Q_{v}$; the state space representation of the SG model is, thus:

$$
\dot{x}(t)=\left(\begin{array}{cccc}
0 & 0 & 0 & \frac{1}{T_{n}} \\
0 & -\frac{1}{T_{h}} & 0 & -\frac{1}{T_{n}} \\
0 & 0 & -\frac{1}{T_{g}} & 0 \\
0 & 0 & 0 & -\frac{1}{\tau}
\end{array}\right) \boldsymbol{x}(t)+\left(\begin{array}{c}
0 \\
0 \\
0 \\
\frac{1}{\tau}
\end{array}\right) u(t)+\left(\begin{array}{c}
-\frac{1}{T_{n}} \\
0 \\
\frac{1+F_{g}}{T_{n}} \\
0
\end{array}\right) d(t)
$$




$$
y(t)=\left(\begin{array}{cccc}
1 & 1 & 1 & 0 \\
\frac{T_{n}}{T_{\text {int }}} & 0 & 0 & \frac{\tau}{T_{\text {int }}}
\end{array}\right) \boldsymbol{x}(t)
$$

The values of the parameters $T_{h}, T_{n}, F_{g}, \tau, T_{g}, T_{i n t}$ change depending on the power $P_{o}$, as shown in Table 1.

Table 1. Parameters of the UTSG model at different power levels [Aubry et al., 2012]

\begin{tabular}{|c|c|c|c|c|c|c|c|}
\hline$P_{o}$ & $0.03 \times P_{n}$ & $0.04 \times P_{n}$ & $0.09 \times P_{n}$ & $0.24 \times P_{n}$ & $0.30 \times P_{n}$ & $0.50 \times P_{n}$ & $P_{n}$ \\
\hline$T_{n}$ & 36 & 56 & 63 & 44 & 40 & 40 & 40 \\
\hline$F_{g}$ & 13 & 18 & 10 & 4 & 4 & 4 & 4 \\
\hline$T_{h}$ & 170 & 56 & 30 & 10 & 8 & 5 & 5 \\
\hline$\tau$ & 10 & 10 & 10 & 30 & 30 & 30 & 30 \\
\hline$T_{g}$ & 10 & 10 & 10 & 10 & 10 & 10 & 10 \\
\hline$T_{\text {int }}$ & 140 & 140 & 140 & 140 & 140 & 140 & 140 \\
\hline
\end{tabular}

The goal of the system is to maintain the SG water level at a reference position $\left(N_{\text {ref }}\right)$ : the SG fails if the $N_{r l}$ rises (falls) above (below) the threshold $N_{\text {high }}\left(N_{l o w}\right)$, in which case automatic reactor or turbine trips are triggered. Indeed, if the $N_{r l}$ exceeds $N_{\text {high }}$, the steam separator and dryer lose their functionality and excessive moisture is carried in $Q_{v}$, degrading the turbine blades profile and the turbine efficiency; if $N_{r l}$ decreases below $N_{l o w}$, insufficient cooling capability of the primary fluid occurs. Similarly, the $W_{r l}$, is relevant for the cooling capability of the primary circuit [Kothare et al., 2000]. Pre-alarms are triggered when $N_{r l}$ exceeds $N_{h l}\left(N_{l l}\right)$ if a small deviation from $N_{\text {ref }}$ occurs or when $N_{r l}$ exceeds $N_{v h}\left(N_{v l}\right)$, when the deviation is large. Set points of $N_{r e f}$ and of $N_{r l}$ depend on $P_{o}$, as shown in Fig. 2, and, thus, also the alarms thresholds depend on $P_{o}$. The $N_{r l}$ set point is low at low $P_{o}$, to partially account for the strong inverse response of $N_{r l}$ [Kothare et al., 2000]; thus, the low level thresholds are more restrictive than the high level thresholds at low $P_{o}$. 

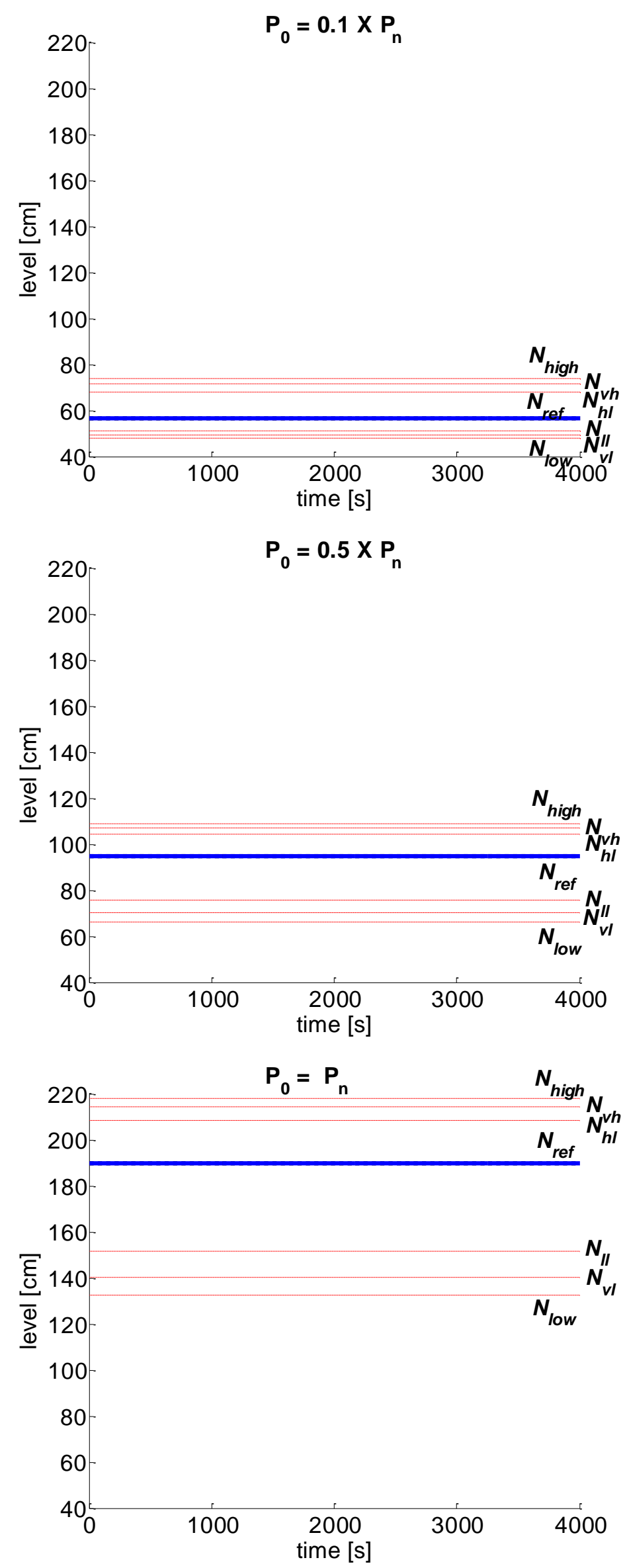

Fig. 2. Set point for $N_{r l}$ at different power rate $P_{o}$ values. 
A dedicated model has been implemented in SIMULINK to simulate the dynamic response of the UTSG at different $P_{o}$ values. Both feedforward and feedback digital control schemes have been adopted. The feedback controller is a PID that provides a flow rate $Q_{\text {pid }}$ resulting from the residuals between $N_{r l}$ and $N_{r e f}$, whereas the feedforward controller operates a safety relief valve that is opened if and only if $N_{r l}$ exceeds the $N_{h l}$, and removes a constant flow safety flow rate $\left(Q_{s f}\right)$. The block diagram representing the SIMULINK model of the SG is shown in Fig. 3: the controlled variable is $N_{r l}$, whereas the control variable is $Q_{e}$.

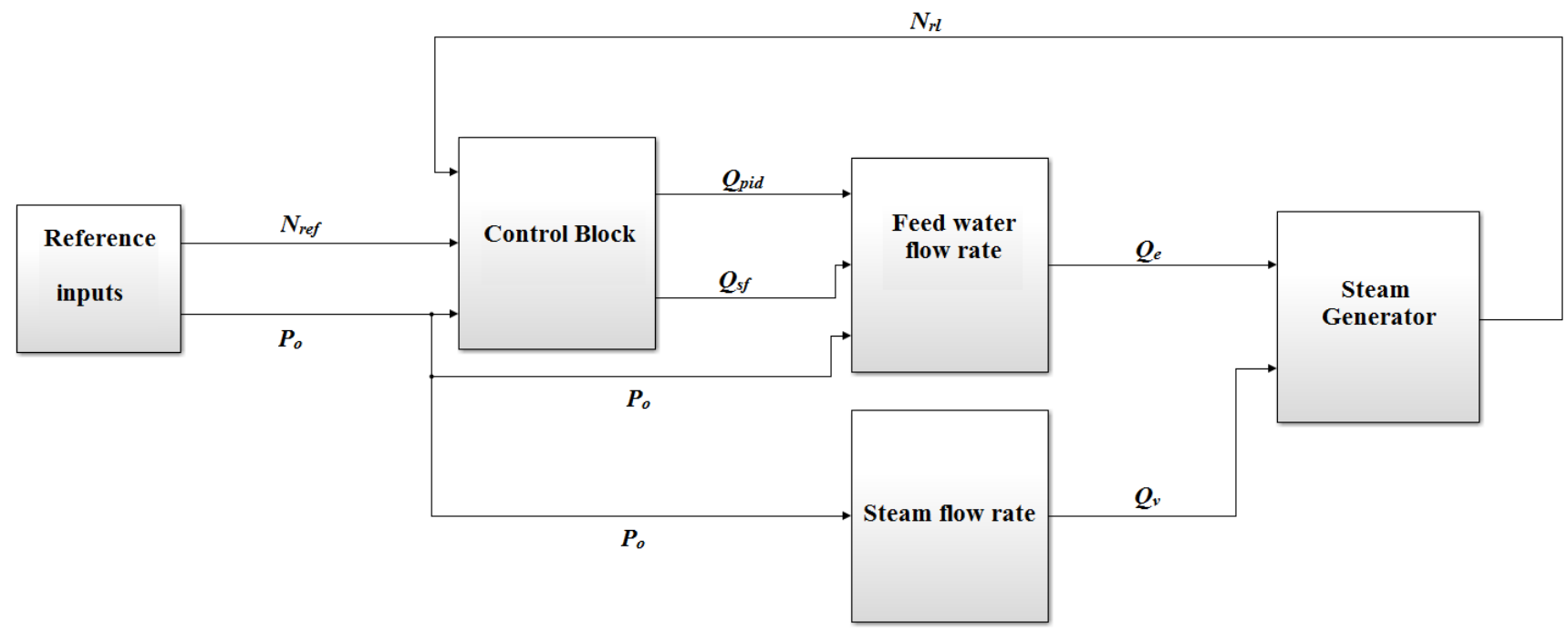

Fig. 3. Block diagram representing the SIMULINK model of the SG.

\subsection{The set of possible failures}

The set of multiple component failures that can occur during the system life are shown in Fig. 4:

1. The outlet steam valve can fail stuck at a random time in $[0,4000]$ (s) in three different positions: i) closed; ii) stuck open at $50 \%$ of the nominal $Q_{v}$ that should be provided at $P_{o}$; iii) stuck open at $150 \%$ of the nominal $Q_{v}$ that should be provided at $P_{o}$.

2. The safety relief valve can fail stuck at a random time in $[0,4000]$ (s), at a uniform random value $Q_{s f}$ in the range $[0.5,50.5](\mathrm{kg} / \mathrm{s})$. 
3. The communication between the sensor that monitors $N_{r l}$ and the PID controller can fail at random times in $[0,4000](\mathrm{s})$, in which case the PID is provided with the same input value of the previous time step.

4. The PID controller can fail stuck at random times in $[0,4000]$ (s), providing a uniform random flow rate $Q_{\text {pid }}$ belonging to $[-18,18] \%$ of the nominal $Q_{e}$ that should be provided at $P_{o}$.

It is worth noticing that in the UTSG there are two PID controllers and, thus, two communications between the sensors measuring $N_{r l}$ and the PIDs (one for high power feedback control and the other for low power feedback control). The selective action of the PIDs depending on $P_{o}$ hides some of the failures. For example, if the power profile of the scenario under investigation is a ramp, both PIDs are called in operation: if anyone (or both) is (are) failed, their fault state is detectable. On the contrary, if we consider scenarios with constant power profile, e.g., low power rate $\left(P_{o}<15 \% P_{n}\right)$, the occurrence of a high power feedback control failure cannot be detected, and, thus, the fault remains hidden.

Choices and hypotheses for modeling the failures (i.e., the mission time, the number and type of faults, the distributions of failure times and magnitudes) have been arbitrarily made with the aim of generating multiple failures in the sequences and capturing the dynamic influence of their order, timing and magnitude. The choice of a mission time $\left(T_{m i s s}\right)$ equal to 4000 (s) has been made, because it is a long enough interval of time to allow the complete development also of slow dynamic accident scenarios. 


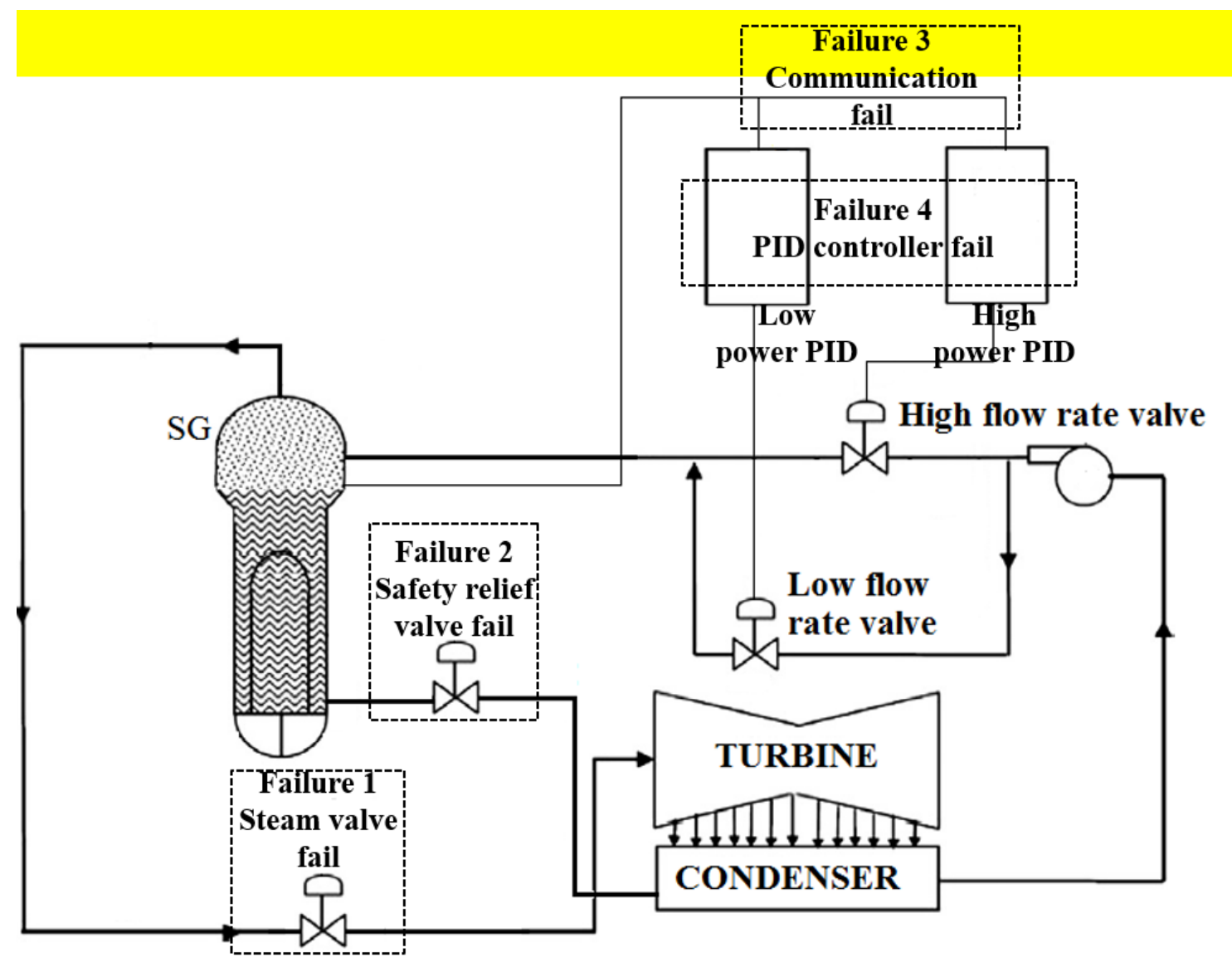

Fig. 4. Sketch of the failures that can be injected into the system

\subsection{The Multistate representation of system dynamics}

For realistically treating the dynamic behavior of the UTSG when component failures occur, we go beyond the binary state representation and adopt a Multiple Value Logic (MVL) [Garibba et al., 1985; Di Maio et al., 2014a] for an approximated description of the continuous time of occurrence of component failures and their magnitude. The MVL allows describing that the components can fail at any (discrete) time (not only the initial time) along the scenario, with different (discrete) magnitudes (not only the most conservative). The discretization of the time and magnitudes values is as follows:

- time discretization: we use the label $t_{m v l}=1, t_{m v l}=2, t_{m v l}=3$ and $t_{m v l}=4$, for failures occurring in the intervals $[0,1000](s),[1001,2000](s),[2001,3000](s),[3001,4000](s)$, respectively; if the label $t_{m v l}=0$, the component does not fail within the time of the whole scenario, $T_{m i s s}$.

- Magnitude discretization: 
- the steam valve magnitude is indicated as 1,2 or 3 for failure states corresponding to stuck at $0 \%$, stuck at $50 \%$ and stuck at $150 \%$ of the $Q_{e}$ value that should be provided at $P_{o}$, respectively; if the steam valve magnitude is indicated as 0 , the component does not fail in $T_{\text {miss }}$;

- the safety relief valve fails with magnitude indicated as 1,2, 3 and 4, if it is stuck between $[0.5,12.6](\mathrm{kg} / \mathrm{s}),(12.6,25.27](\mathrm{kg} / \mathrm{s}),(25.27,37.91](\mathrm{kg} / \mathrm{s})$ and $(37.91,50.5]$ $(\mathrm{kg} / \mathrm{s})$, respectively; if the safety relief valve magnitude is indicated as 0 , the component does not fail in $T_{\text {miss }}$;

- the communication between the sensor measuring $N_{r l}$ and the PID controller is labelled 0 if the communication works, 1 otherwise;

- the PID controller failure magnitude range is discretized into 8 equally spaced magnitude intervals, labelled from 1 to 8 , representative of failure states corresponding to discrete intervals of output value belonging to $[-18,18] \%$ of the $Q_{e}$ value that should be provided at $P_{o}$; if the PID controller magnitude is labelled as 0 , the component does not fail in $T_{\text {miss }}$.

The values of time, magnitude and order of failure occurrence for each component are included into a sequence vector that represents a scenario. As an example, the sequence vector of Fig. 5 represents a scenario where: the steam valve fails stuck at its maximum allowable value at a time in [3001, 4000] (s) and it is the third event occurring along the sequence; the safety relief valve fails first in [0, 1000] (s), with a magnitude belonging to $[0.5,12.6](\mathrm{kg} / \mathrm{s})$; the communication between the sensor measuring $N_{r l}$ and the PID controller is the second failure event in the sequence and occurs in [2001, 3000] (s); finally, the PID controller fails stuck in [3001, 4000] (s), with a magnitude belonging to $[6,10] \%$ of the $Q_{e}$ value that should be provided at $P_{o}$.

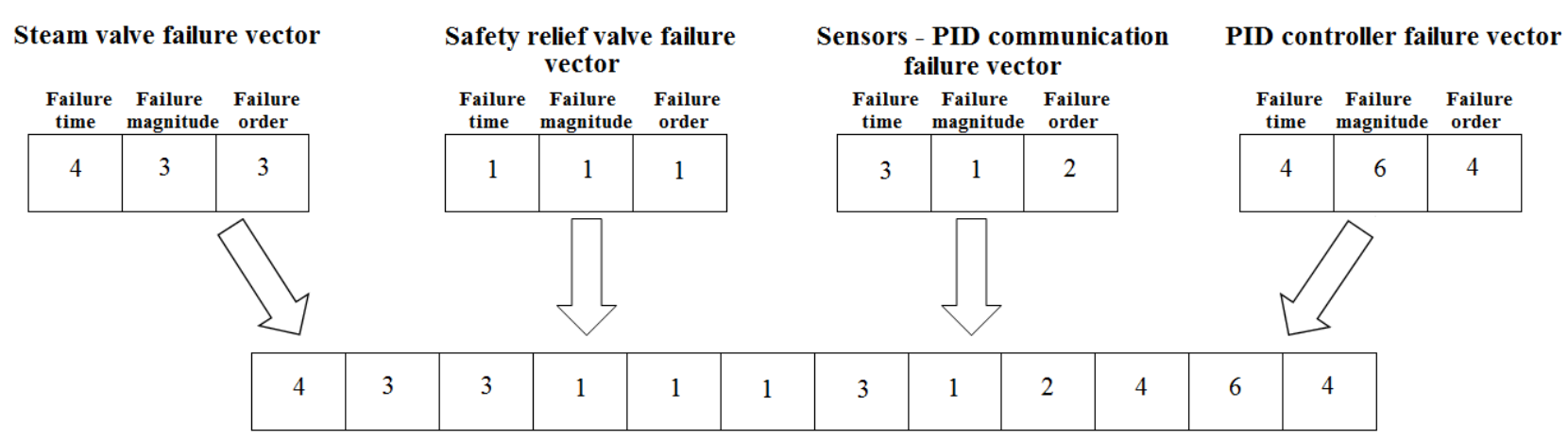

Fig. 5. Sequence vector representing a scenario. 
The number of possible sequence vectors that arise from the MVL discretization are 100509, each one evolving towards either safe or faulty conditions. To investigate this, a Monte Carlo-driven fault injection engine is used to sample combinations of discrete times and discrete magnitudes of components failures.

The (dynamic) analysis has been performed with respect to the two constant power scenarios, $5 \% P_{n}$ (low power level) and $80 \% P_{n}$ (high power level). The system configurations considered are listed in Table 2.

Table 2. System configurations

\begin{tabular}{|c|c|c|c|c|}
\hline $\begin{array}{c}\text { System } \\
\text { configurations }\end{array}$ & $\begin{array}{c}\text { Failure of the } \\
\text { Outlet Steam } \\
\text { Valve }\end{array}$ & $\begin{array}{l}\text { Failure of the } \\
\text { Safety Relief } \\
\text { Valve }\end{array}$ & $\begin{array}{l}\text { Level sensor- } \\
\text { PID controller } \\
\text { communication } \\
\text { interruption }\end{array}$ & $\begin{array}{c}\text { Failure of } \\
\text { the PID } \\
\text { controller }\end{array}$ \\
\hline 1 & - & - & - & - \\
\hline 2 & $\mathbf{X}$ & - & - & - \\
\hline 3 & - & $\mathbf{X}$ & - & - \\
\hline 4 & - & - & $\mathbf{X}$ & - \\
\hline 5 & - & - & - & $\mathbf{X}$ \\
\hline 6 & $\mathbf{X}$ & $\mathbf{X}$ & - & - \\
\hline 7 & $\mathbf{X}$ & - & $\mathbf{X}$ & - \\
\hline 8 & $\mathbf{X}$ & - & - & $\mathbf{X}$ \\
\hline 9 & - & $\mathbf{X}$ & $\mathbf{X}$ & - \\
\hline 10 & - & $\mathbf{X}$ & - & $\mathbf{X}$ \\
\hline 11 & - & - & $\mathbf{X}$ & $\mathbf{X}$ \\
\hline 12 & $\mathbf{X}$ & $\mathbf{X}$ & $\mathbf{X}$ & - \\
\hline 13 & $\mathbf{X}$ & $\mathbf{X}$ & - & $\mathbf{X}$ \\
\hline 14 & $\mathbf{X}$ & - & $\mathbf{X}$ & $\mathbf{X}$ \\
\hline 15 & - & $\mathbf{X}$ & $\mathbf{X}$ & $\mathbf{X}$ \\
\hline 16 & $\mathbf{X}$ & $\mathbf{X}$ & $\mathbf{X}$ & $\mathbf{X}$ \\
\hline
\end{tabular}

The dynamic analysis shows that the same combination of components failures does not unequivocally lead to only one system end state but, rather, it depends on when the failures occur and with what magnitude. This is shown in Fig. 6, where the frequencies of occurrence of the three system end states ("High", "Safe" and "Low") are plotted for the 16 dynamic system configurations of Table 2. 

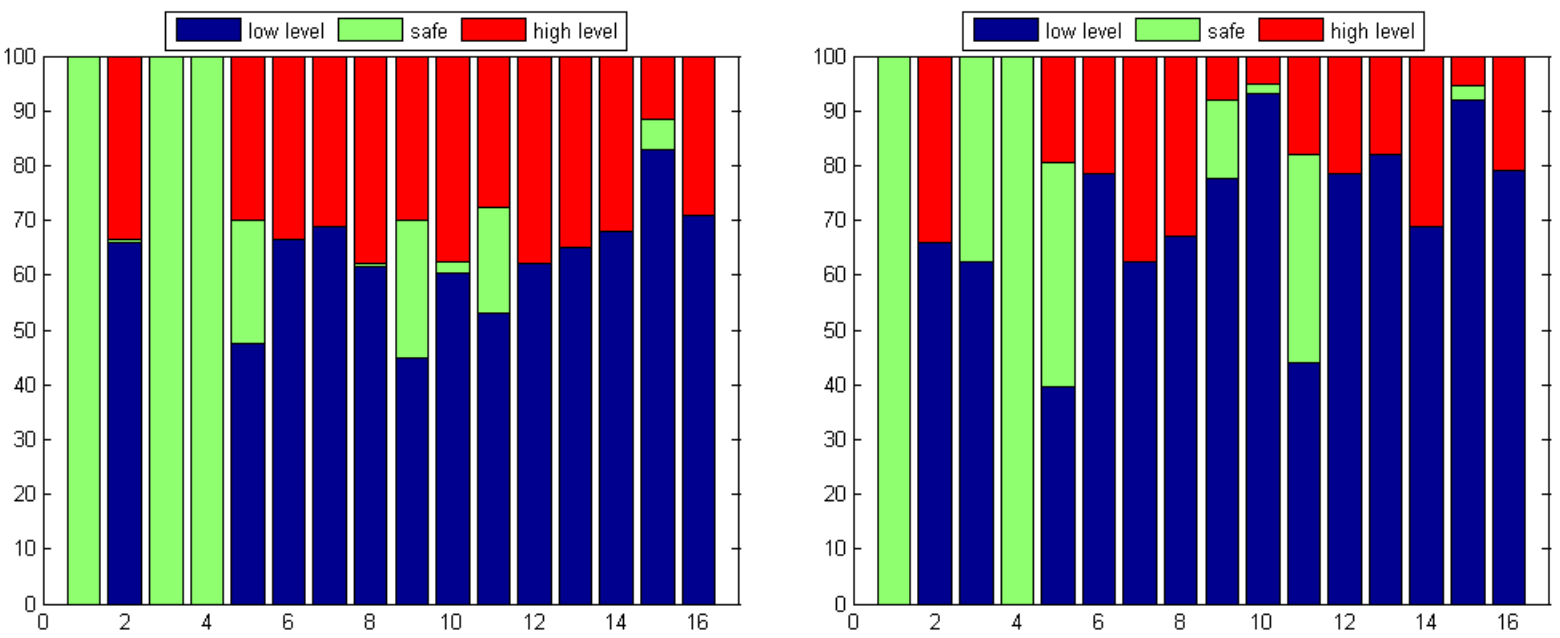

Fig. 6. Histograms for high power level (a) and low power level (b) of the frequencies of occurrence of the end states for each of the 16 system configurations of Table 2, simulated by sampling discrete failure times and magnitudes of components failures.

Fig. 7 shows that, at high power operation, the timing of the events is quite important, because with the same system configuration but different times of failure occurrences, the system end state changes. Specifically, in Fig. 7 (a), the safety valve fails stuck at $100 \%$ of $Q_{s f}$ after 1020 seconds and the communication between the sensor measuring $N_{r l}$ and the PID controller fails at:

- 1052 seconds (solid line).

- 1063 seconds (dashed-dotted line).

The two scenarios lead to low and high failure modes, respectively, whereas they would be considered as Minimal Cuts Sets (MCS) in a static reliability analysis presented in Appendix 1.

Fig. 7 (b) shows the effects of different failures magnitudes on the system end state: the safety relief valve fails stuck in its maximum position at 2000 seconds, the communication fails at 2010 seconds and the PID controller fails at 2020 seconds with two different magnitudes:

- Magnitude equal to $13 \%$ of the nominal $Q_{e}$ value that should be provided at $80 \% P_{n}$ (dasheddotted line).

- Magnitude equals to $12 \%$ of the nominal $Q_{e}$ value that should be provided at $80 \% P_{n}$ (solid line). 


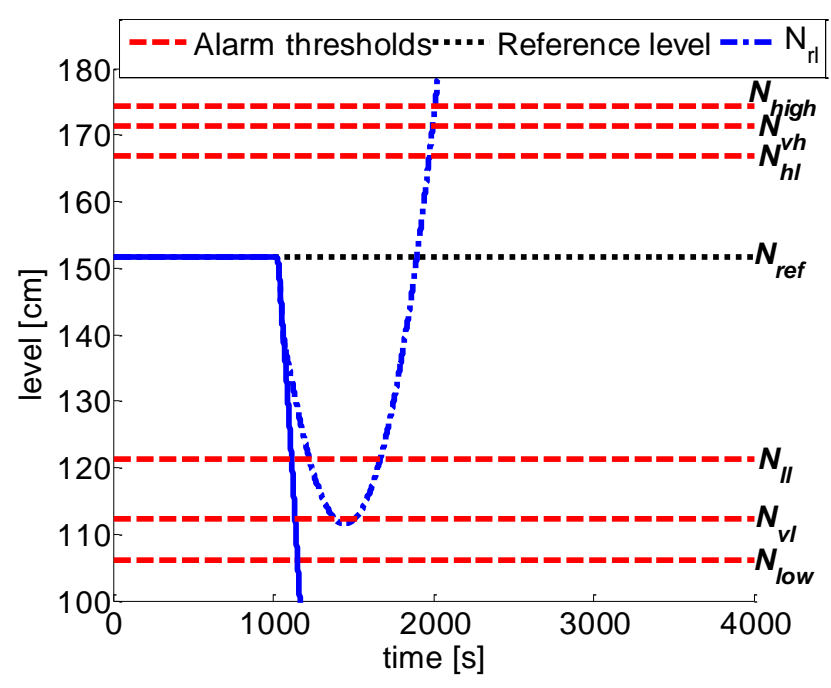

(a)

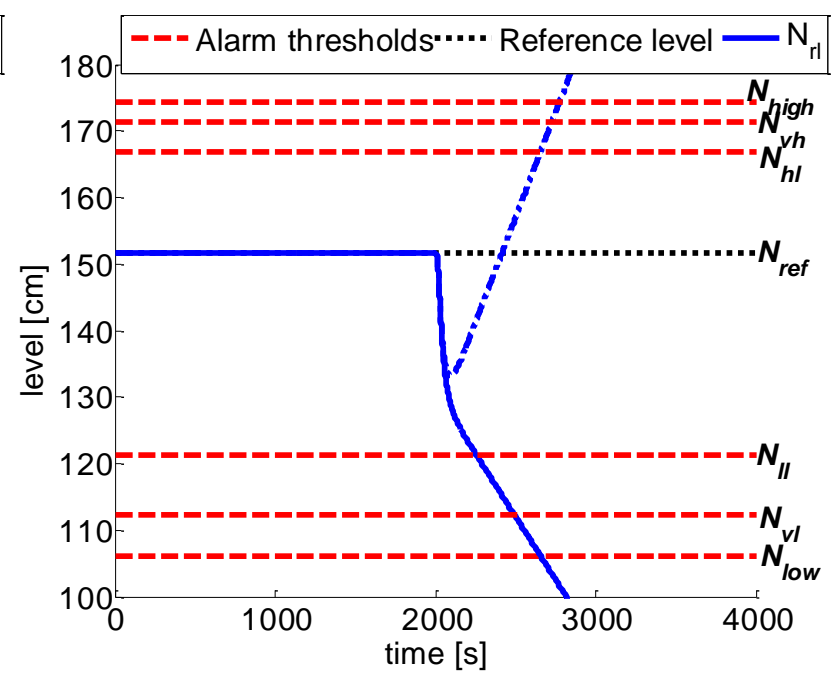

(b)

Fig. 7. Example of dynamic system behavior at $80 \% P_{n}$.

The low power scenarios also present dynamic effects, as shown in Fig. 8. In particular, Fig. 8 (a) shows the effects of the timing on the system end state: the safety relief valve fails stuck at $Q_{s f}=50.5$ $(\mathrm{kg} / \mathrm{s})$ at 1005 (s) and the steam output valve fails stuck at $150 \%$ of the nominal $Q_{v}$ value that should be provided at $5 \% P_{n}$ at:

- 1046 seconds (dashed-dotted line).

- 1047 seconds (solid line).

Fig. 8 (b) shows the effects of the order of components failure occurrence on the system end state: the safety relief valve fails stuck at $Q_{s f}=50.5(\mathrm{~kg} / \mathrm{s})$ and the PID controller fails stuck at its minimum allowable value:

- The PID controller failure is the first failure event along the sequence of events (dashed-dotted line).

- The safety relief valve failure is the first failure event along the sequence of events (solid line). 


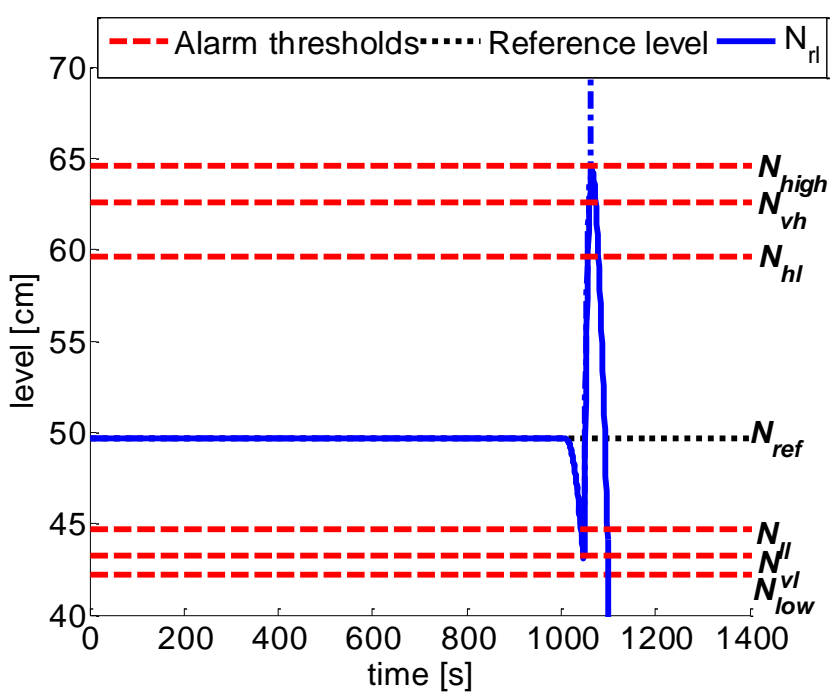

(a)

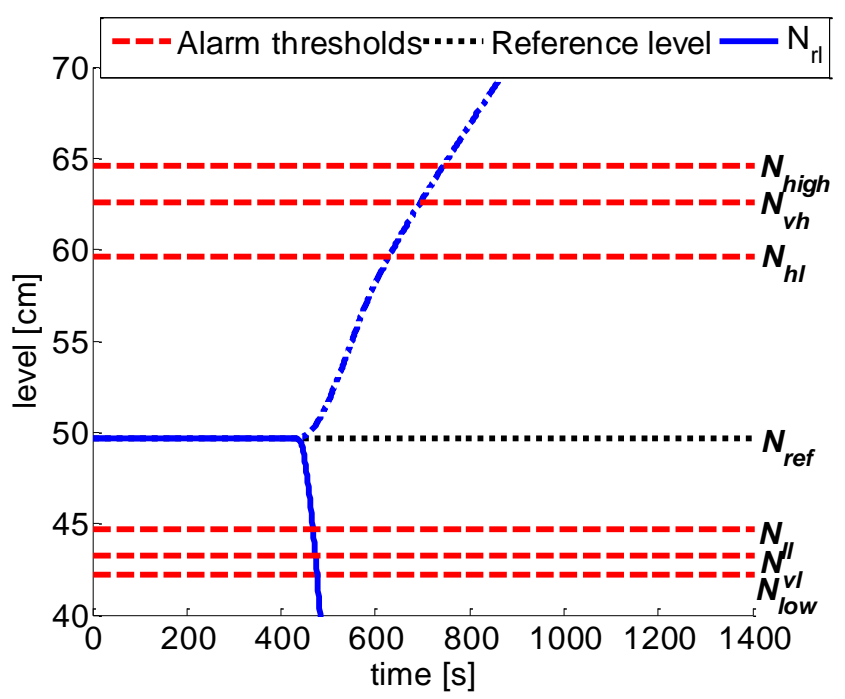

(b)

Fig. 8. Example of dynamic system behavior at $5 \% P_{n}$.

Hereafter, without loss of generality, among the system configurations of Table 2, we focus only on the classification of the PIs and Near Misses of the high level failure mode at high power level $\left(P_{o}=\right.$ $\left.80 \% P_{n}\right)$.

\section{Near Misses identification}

The Near Misses identification is here treated as a classification problem, in which Near Misses are sorted out from the safe scenarios, among the whole set of accidental transients simulated. In practice, the PIs are first identified among the whole set of 100509 possible scenarios and, then, the Near Misses are separated out among the remaining safe scenarios.

\subsection{Prime Implicants identification}

A PI is a set of variables that represents a minimal combination of accident component failures necessary for system failure and cannot be covered by a more reduced implicant [Quine, 1952; Di Maio et al., 2014a]. Note that in our case the "PIs" identification task may consider non-coherent structure functions, for which both failed and working states of the same components can lead the system to failure. In such circumstances, traditional methods, e.g. based on minimal cut sets analysis, 
cannot be applied, whereas dynamic reliability methods need to be applied for the identification of the PIs [Di Maio et al., 2013; Di Maio et al., 2014b].

The PIs identification among the whole set of 100509 possible scenarios is performed by means of the visual interactive method presented in [Di Maio et al., 2014b]. The basic idea it relies on is that PIs are those scenarios with as few as possible events that are capable of leading the system into a failure state [Rocco et al., 2004]; then, we first select as most important feature for the PIs identification the literal cost of the sequence vector (i.e., the number of components whose behavior is specified in the accident sequence) and then, the accident sequences associated with the lowest literal cost are selected and stored as PIs. In fact, these are the most reduced sequences (i.e., with least number of events) that cannot be covered by any other implicant, and, thus, these are PIs by definition. The selected PIs, and the implicants covered by them, are deleted from the set of implicants and, the procedure is repeated for the remaining implicants until all are covered. By so doing, 1255 PIs are identified for the high level failure mode, covering 36128 minterms. The total computational time approximately required for the identification of the PIs is 780 (s) on an Intel ${ }^{\circledR}$ Core ${ }^{\mathrm{TM}} 2$ Duo T9300 CPU@2.50 GHz.

\subsection{The "forward" classification}

Once the (1255) PIs for the SG high level failure mode have been identified, they are removed from the set of all possible scenarios, which is left with 64381 safe scenarios. For the identification of Near Misses among these, we resort to their definition as sequences of failure events that indeed keep the system in a safe condition, but endangered (i.e., a quasi-fault system state). To this aim, we introduce a risk-based characterization of these remaining scenarios, calculating their associated risk, at each time instant $t$, as [Zadakbar et al., 2013]:

$$
\operatorname{Risk}(t)=p(t) \times c(t)
$$

where $p(t)$ is the probability that at time $t$ the scenario can lead the system into an accidental scenario and $c(t)$ is the consequence that the developing scenario is predicted to cause to the system.

In this view, we build a functional relationship such that $p$ increases as $N_{r l}$ moves further away from the reference level $N_{r e f}$, in a way that $p=0$ if $N_{r l}$ is equal to $N_{r e f}$ and $p=1$ if $N_{r l}$ reaches $N_{h i g h}$. 
Such relationship is given in Eq. (9) below, assuming that scenarios whose $N_{r l}(t)$ approaches $N_{\text {high }}$ are more prone to failure than those with $N_{r l}(t)$ close to $N_{r e f}$, i.e., Eq. (9) "filters-out" (i.e., neglects) scenarios whose $N_{r l}(t)$ is close to $N_{r e f}$ and "mines" (i.e., weighs more) scenarios whose $N_{r l}(t)$ is close to $N_{\text {high }}$ :

$$
p(t)=\varphi\left(\frac{N_{r l}(t)-(\mu+5 \sigma)}{\sigma}\right)=\int_{N_{r e f}}^{N_{r l}(t)} \frac{1}{\sqrt{2 \pi} \sigma} e^{\frac{\left(N_{r l}(t)-(\mu+5 \sigma)\right)^{2}}{2 \sigma^{2}}} d N_{r l}
$$

where $\varphi$ is the cumulative probability function of the Gaussian distribution with mean $\mu=N_{r e f}$, and standard deviation $\sigma=\frac{N_{\text {high }}-N_{\text {ref }}}{5}$. Fig. 9 shows the trend of $p(t)$.

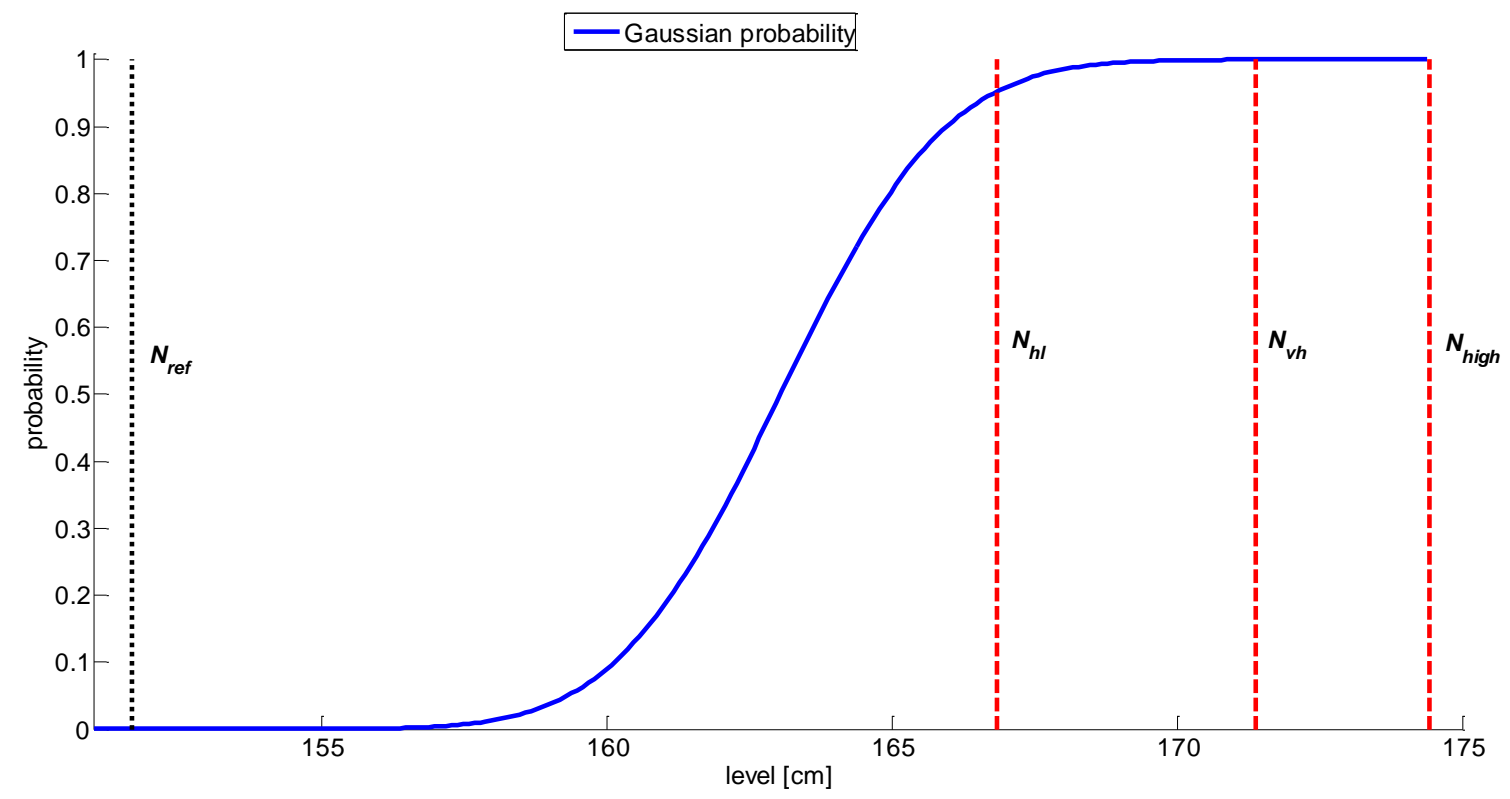

Fig. 9. Probability function $p(t)$ for the definition of risk.

The consequence $c(t)$ of a scenario increases as $N_{r l}$ approaches the failure threshold $N_{\text {high }}, c(t)$ can be calculated at time $t$ as [Zadakbar et al., 2013]:

$$
c(t)=A^{\frac{N R L(t)-(\mu+3 \sigma)}{N R L(t)-\mu}}
$$


where $A$ is the intensity coefficient that accounts for the closeness of $N_{r l}$ to the thresholds $N_{h l}, N_{v h}$ and $N_{\text {high }}$, and for the exceedance time between the first event of the failure sequence (hereafter called Initiating Event (IE)) and the time of exceeding the threshold: the shorter this time, the more critical the scenario. Thus, $A$ is larger the faster and closer $N_{r l}$ approaches a threshold; we assume: $A=100$ (No consequences) if no threshold is exceeded; $A=200$ (Low consequences) if $N_{r l}$ exceeds $N_{h l}$ after at least 2001 (s) from IE or if $N_{r l}$ exceeds $N_{v h}$ after at least 3001 (s) from IE; $A=300$ (Medium consequences) if $N_{r l}$ exceeds $N_{h l}$ within 2000 (s) from IE, if $N_{r l}$ exceeds $N_{v h}$ and the elapsed time is in $[1001,3000]$ (s), and if $N_{r l}$ exceeds $N_{\text {high }}$ after at least 2001(s) from IE; $A=400$ (Catastrophic consequences) if $N_{r l}$ exceeds $N_{v h}$ within 1000 (s) from IE or if $N_{r l}$ exceeds $N_{h i g h}$ and the elapsed time from IE is in $[1,2000]$ (s). A matrix representation of the intensity coefficient is shown in Fig. 10.

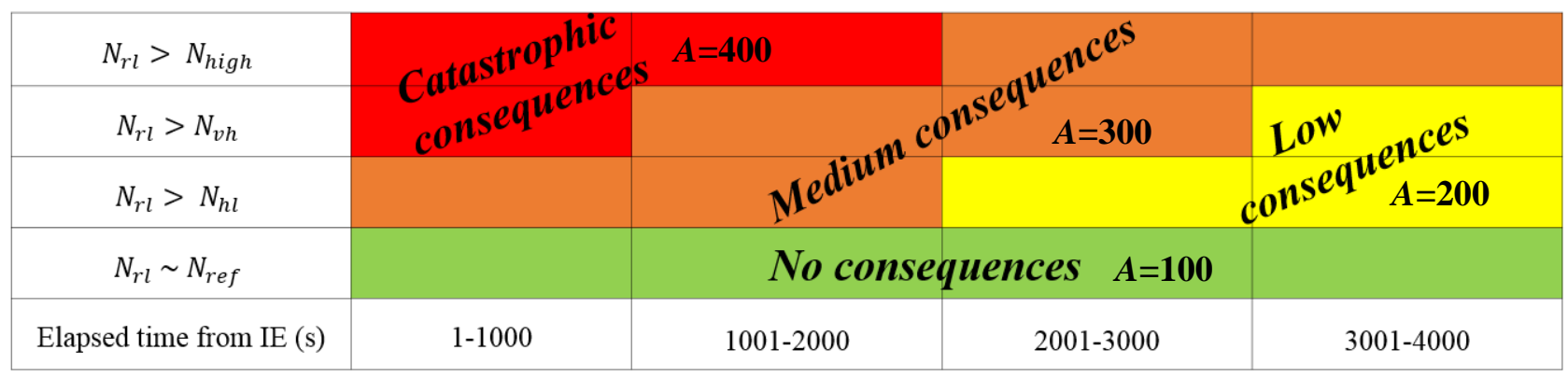

Fig. 10. Matrix representation of the intensity coefficient $A$.

By so doing, the available 64381 remaining safe scenarios are fully described at each time instant $t$ $=1,2 \ldots . .4000[\mathrm{~s}]$ by their values of probability $p(t)$, consequence $c(t)$ and overall risk $r(t)$. An example of the $p(t), c(t), r(t)$ evolutions for two generic trends of $N_{r l}(t)$ is shown in Fig. 11. More specifically, the $N_{r l}(t)$ behaviors represented in Fig. 11 (first row) are due to:

- solid line: the PID controller fails at 100 (s) with magnitude 4 and the safety relief valve fails at 190 (s) with magnitude 2;

- dashed-dotted line: the safety relief valve fails at 100 (s) with magnitude 1, the communication between the sensor measuring $N_{r l}$ and the PID controller is interrupted at 136 (s) and the PID controller fails at 3917 (s) with magnitude 5; 
It is worth analysing the behavior of $p(t), c(t)$, and, thus, $r(t)$ considered (Fig. 11, second, third and fourth row, respectively): all three abovementioned functions increase as $N_{r l}(t)$ moves further away from $N_{r e f}$ and decrease as $N_{r l}(t)$ approach $N_{r e f}$. The steps shown in the consequences and risk plots (around 800 [s] for the solid line scenario and around 3500[s] for the dashed-dotted line scenario) are due to the change of the discrete consequence intensity coefficient $A$ along the scenarios. The solid line scenario is faster than the dashed-dotted line scenario (upper plot) and, thus, the value of the parameters $A$ for the former scenario is 400 (Catastrophic consequences, see Fig. 10), due to the fact that $N_{r l}(t)$ exceeds $N_{v h}$ within 1000 (s), whereas, $A=300$ (Medium consequences, see Fig. 10) for the dashed-dotted scenarios, because $N_{r l}(t)$ exceeds $N_{v h}$ within [1001, 3000] (s). Thus, the solid line scenario is more abrupt in its development towards failure and expected to have more catastrophic consequences, and, thus, more overall risk, than the dashed-dotted scenario, because the time between IE and the exceedance of $N_{v h}$ is shorter (i.e, less grace time).

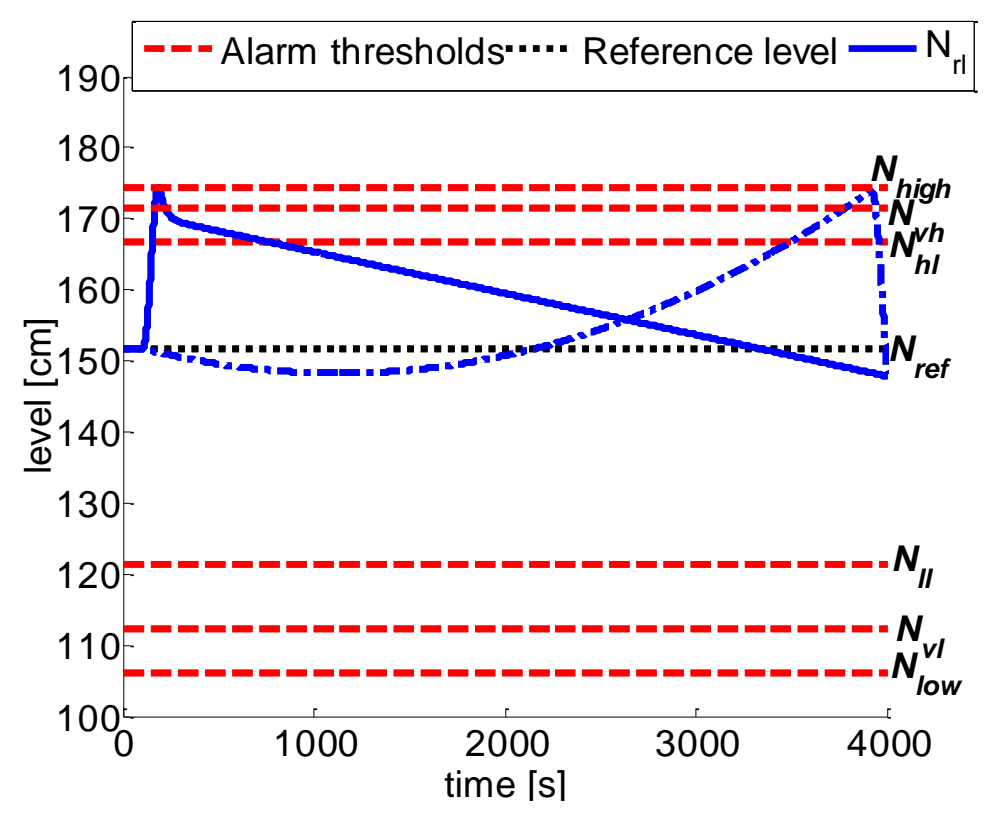



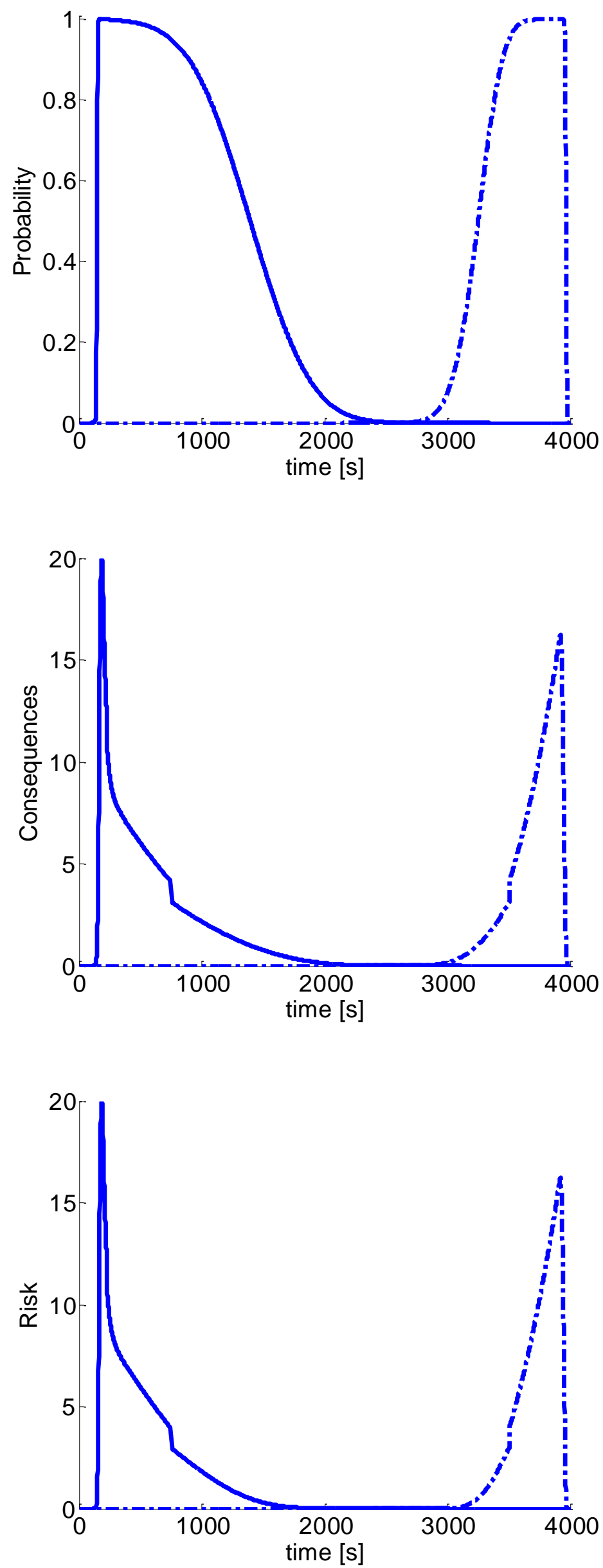
Fig. 11. Probability $p(t)$, consequences $c(t)$ and risk $r(t)$ for two sequences of events.

\subsubsection{Features selection}

The identification of the Near Misses is treated as an unsupervised classification problem and addressed by clustering, where i) the number of clusters is unknown and ii) the features that enable the best clustering according to the risk-based characteristic profiles of $p(t), c(t)$ and $r(t)$ of the accidental scenarios are unknown. Unsupervised clustering, thus, entails identifying the number $K$ of clusters in which similar scenarios can be grouped according to similar values of some scenario features. To do this, from the profiles $p(t), c(t)$ and $r(t)$, we extract some statistical indicators as features [Zio et al., 2012b]:

1. Mean value

$$
\mu_{n}=\frac{1}{T_{\text {miss }}} \sum_{t=1}^{N} n(t)
$$

2. Peak value

$$
\max =\max _{t=1,2 \ldots . T_{\text {miss }}} n(t)
$$

3. Standard deviation

$$
\sigma_{n}=\sqrt{\frac{1}{T_{m i s s}-1} \sum_{t=1}^{T_{m i s s}}(n(t)-\mu)^{2}}
$$

4. Root mean square

$$
\mathrm{RMS}=\sqrt{\frac{1}{T_{\text {miss }}} \sum_{t=1}^{T_{\text {miss }}}(n(t))^{2}}
$$

5. Skewness

$$
S K=\frac{\sum_{t=1}^{T_{m i s s}}(n(t)-\mu)^{3}}{T_{m i s s}-1}
$$

6. Kurtosis

$$
K U=\frac{\sum_{t=1}^{T_{m i s s}}(n(t)-\mu)^{4}}{T_{m i s s}-1}
$$

where $n(t)$ is alternatively equal to $p(t), c(t)$ and $r(t)$ and, thus, the total number of features is equal to $6 \times 3=18$. Among these 18 available features, we search for those that are optimal for clustering the 64381 scenarios in Near Misses and safe scenarios.

We resort to a wrapper framework [Kohavi et al., 1997; Baraldi et al., 2014], whereby a Modified Binary Differential Evolution (MBDE) search engine [Wang et al., 2010; Di Maio et al., 2013] searches candidate groups of features sets that are fed to a K-means clustering algorithm [MacQueen, 1967]; eventually, the wrapper evolves so that among these candidate groups, the group retained is that which makes the K-means clustering algorithm perform best (most compact and separate 
clusters). The idea behind the wrapper approach is shown in Fig. 12. During the features search by MBDE, the K-means clustering is run on the $N=0.80 \times 64381=51505$ (training) safe scenarios with sets of features $(F)$ that are randomly selected by the MBDE algorithm. The optimal number $(K)$ of clusters is also unknown and it is determined by looking at the clustering performance obtained by the K-means with reference to the Calinski-Harabasz $(\mathrm{CH})$ index [Calinski et al., 1974], which accounts for the ratio of the overall between-cluster variance (separation) and the overall withincluster variance (compactness). The search proceeds iteratively until the $\mathrm{CH}$ index is maximised and the number of clusters $K$ is fixed. Then, the results of the wrapper algorithm are evaluated on an independent test set $\left(T_{\text {set }}\right)$, i.e., the $0.2 \times 643281=12876$ safe scenarios that have been left out during the training phase.

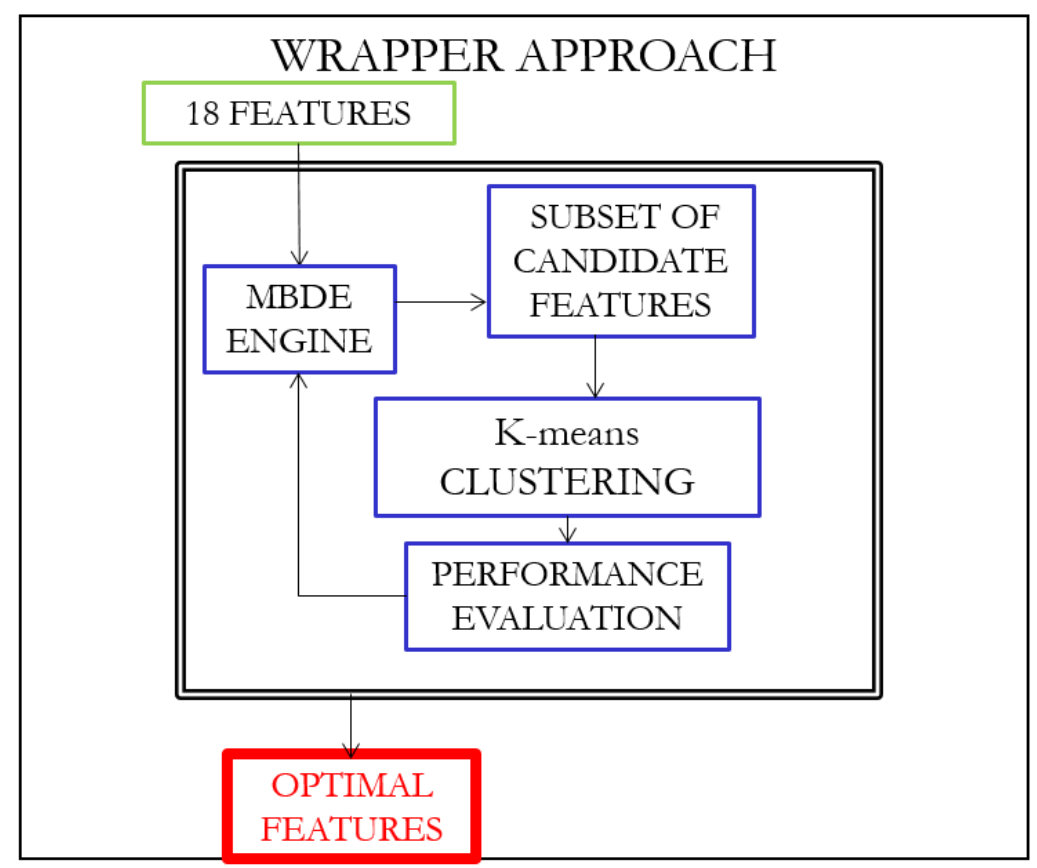

Fig. 12. Wrapper approach for optimal feature subset selection based on a MBDE optimization algorithm and a K-means classifier.

The $\mathrm{CH}$ index for a number $K$ of clusters, $k=1,2, \ldots, K$ is equal to [Calinski et al., 1974]:

$$
C H=\frac{S S_{b}}{S S_{w}} \times \frac{(N \times F)-K}{K-1}
$$


where $S S_{b}$ is the overall between-cluster variance,

$$
S S_{b}=\sum_{k=1}^{K} n_{k}\left\|m_{k}-m\right\|^{2}
$$

and $S S_{w}$ is the overall within-cluster variance,

$$
S S_{w}=\sum_{k=1}^{K} \sum_{x \in k}\left\|x_{c}-m_{k}\right\|^{2}
$$

where, $n_{k}$ is the number of scenarios $x_{c}$ assigned to the $k$-th cluster, $m_{k}$ is the centroid of the $k$-th cluster, i.e., the mean of the selected features belonging to the $k$-th cluster, $m$ is the mean of the selected features, and $\left\|m_{k}-m\right\|^{2}$ and $\left\|x_{c}-m_{k}\right\|^{2}$ are the $\mathrm{L}^{2}$ norms, i.e. Euclidean distances, between the two vectors.

The optimal features selection provides as best features: the standard deviation of $c(t)$, the standard deviation of $r(t)$ and the root mean square of $r(t)$; the best performance is obtained with $\mathrm{CH}=9.35 \mathrm{e}+04$ and $K=5$.

\subsubsection{The clustering results}

The $K=5$ obtained clusters of the safe scenarios are shown in Fig. 13 with reference to the features of mean risk $\left(\mu_{\text {risk }}\right)$ and time elapsed from the instant $t_{\text {risk }}$ at which $r(t)$ starts to deviate from zero, i.e., the time interval during which the system is exposed to risk. The rationale behind this choice is that the larger $\mu_{\text {risk }}$ and the longer $t_{\text {risk }}$, the more dangerous the scenarios. In Fig. 13, clusters 3, 4, 5 (triangles, crosses and squares, respectively) are well separated, i.e., the low level risk scenarios clusters are widened by the adoption of Eq. (9) for the quantification of the risk profile $r(t)$. It is possible to distinguish the scenarios having the lowest risk level from the scenarios having low risk level, and, thus, the highest risk scenarios are well separated from the lower risk scenarios. The good performance obtained when Eq. (9) is adopted instead of other $p(t)$ profiles, e.g., linear probability 
function $\left(p(t) \alpha N_{r l}(t)\right)$ that would give the same importance to any level $N_{r l}$, for the quantification of the risk profile $r(t)$ is due to the fact that Eq. (9) "filters-out" (i.e., neglects) scenarios whose $N_{r l}(t)$ is close to $N_{\text {ref }}$ and "mines" (i.e., weighs more) scenarios whose $N_{r l}(t)$ is close to $N_{h i g h}$ : the 332 circles in Fig. 13 (listed in Appendix 2) can, thus, be considered the Near Misses scenarios, i.e., scenarios that incidentally keep the system into safe state, although in endangered and insecure, operational conditions.

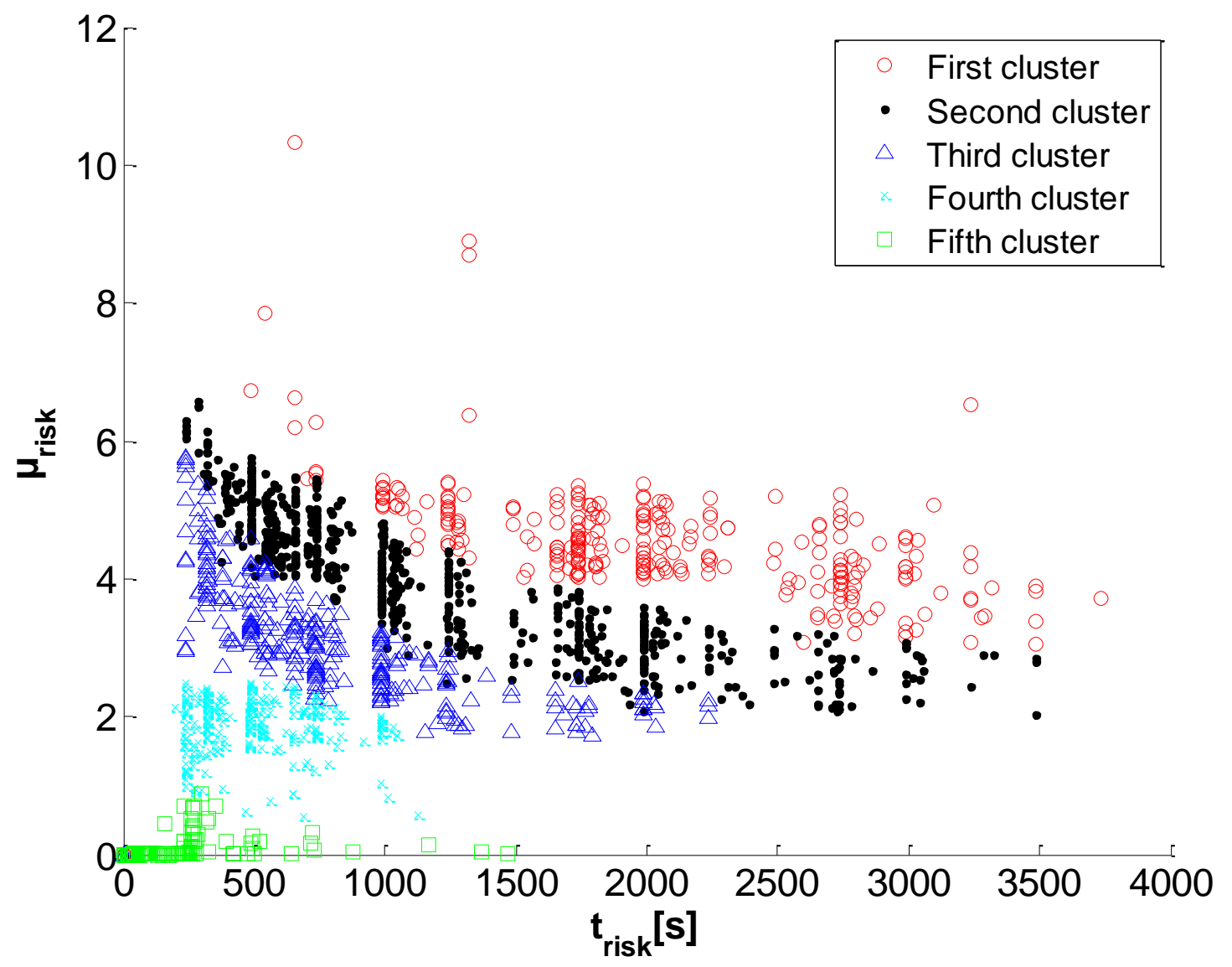

Fig. 13. Clustering results.

\subsection{The "backward" approach}

Once the Near Misses for the SG high level failure mode have been identified by clustering, we can search for similarities among them in terms of their Multiple Value sequences, i.e., order and timing 
of event occurrences and deterministic process variables values. This "backward" approach can lead us to finding the minimum conditions, i.e., minimum $\mu_{\text {risk }}$ and minimum $t_{r i s k}$, that lead the system into a quasi-fault state. The problem can be framed as a Multi-Objective optimization Problem (MOP) [Deb et al., 2002] that looks for the set of scenarios $\overline{\boldsymbol{x}}$ that to dominate any other scenarios with respect to the fitness function $f$ :

$$
f(x)=\left[f_{1}\left(\mu_{\text {risk }}\right), f_{2}\left(t_{\text {risk }}\right)\right]
$$

where $f_{1}$ and $f_{2}$ are the objectives functions of the defined MOP, i.e., minimum $\mu_{\text {risk }}$ and $t_{\text {risk }}$, respectively. The solution of the MOP of Eq. (14) is the Pareto set shown in Fig. 14, where 12 solutions are plotted (squares lined by continuous line) and listed in Table 3. These scenarios $\overline{\boldsymbol{x}}$ of minimum $f(x)$ are expected to cover all failure of Near Misses scenarios cluster.

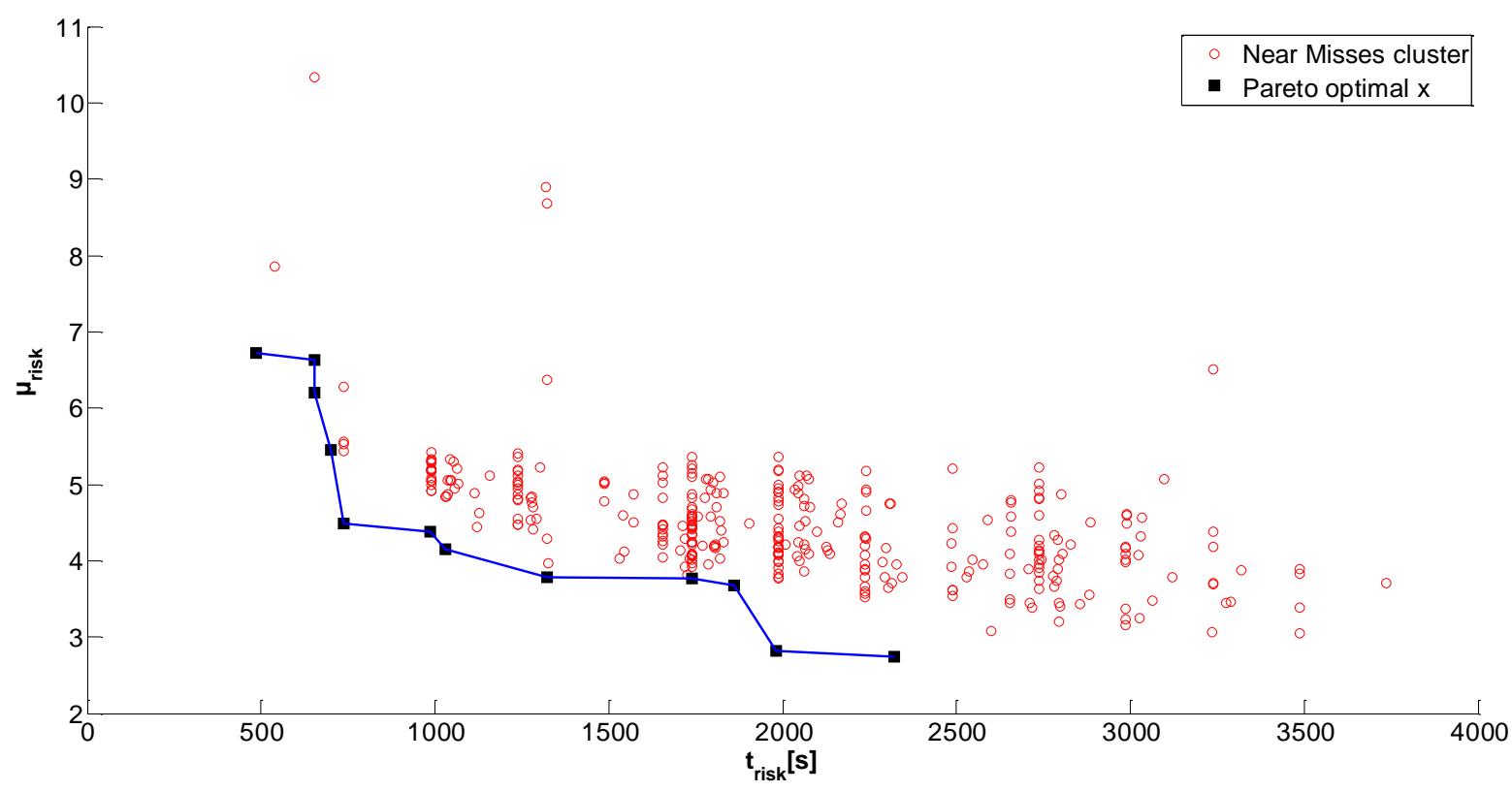

Fig. 14. Pareto front for the cluster of Near Misses 
Table 3. List of the Pareto-optimal $\overline{\boldsymbol{x}}$ sequence vectors.

\begin{tabular}{|c|c|c|c|c|c|c|c|c|c|c|c|c|}
\hline$\overline{\boldsymbol{X}}$ & $\begin{array}{c}\text { Steam } \\
\text { valve } \\
\text { failure } \\
\text { time }\end{array}$ & $\begin{array}{c}\text { Steam } \\
\text { valve } \\
\text { failure } \\
\text { magnitude }\end{array}$ & $\begin{array}{c}\text { Steam } \\
\text { valve } \\
\text { failure } \\
\text { order }\end{array}$ & $\begin{array}{c}\text { Safety } \\
\text { valve } \\
\text { failure } \\
\text { time }\end{array}$ & $\begin{array}{c}\text { Safety } \\
\text { valve } \\
\text { failure } \\
\text { magnitude }\end{array}$ & $\begin{array}{c}\text { Safety } \\
\text { valve } \\
\text { failure } \\
\text { order }\end{array}$ & $\begin{array}{c}\text { Sensor- } \\
\text { PID } \\
\text { failure } \\
\text { time }\end{array}$ & $\begin{array}{c}\text { Sensor- } \\
\text { PID } \\
\text { failure } \\
\text { magnitude }\end{array}$ & $\begin{array}{c}\text { Sensor- } \\
\text { PID } \\
\text { failure } \\
\text { order }\end{array}$ & $\begin{array}{c}\text { PID } \\
\text { failure } \\
\text { time }\end{array}$ & $\begin{array}{c}\text { PID } \\
\text { failure } \\
\text { magnitude }\end{array}$ & $\begin{array}{c}\text { PID } \\
\text { failure } \\
\text { order }\end{array}$ \\
\hline 1. & 0 & 0 & 0 & 2 & 3 & 1 & 4 & 1 & 2 & 4 & 3 & 3 \\
\hline $\mathbf{2 .}$ & 3 & 1 & 4 & 1 & 2 & 3 & 1 & 1 & 2 & 1 & 4 & 1 \\
\hline 3. & 3 & 1 & 4 & 2 & 2 & 3 & 2 & 1 & 1 & 2 & 4 & 2 \\
\hline 4. & 3 & 1 & 3 & 2 & 2 & 2 & 4 & 1 & 4 & 1 & 4 & 1 \\
\hline $\mathbf{5 .}$ & 3 & 1 & 4 & 3 & 3 & 3 & 2 & 1 & 2 & 1 & 4 & 1 \\
\hline $\mathbf{6 .}$ & 3 & 2 & 4 & 1 & 2 & 2 & 1 & 1 & 3 & 1 & 4 & 1 \\
\hline 7. & 3 & 2 & 4 & 2 & 3 & 1 & 2 & 1 & 2 & 3 & 3 & 3 \\
\hline $\mathbf{8 .}$ & 4 & 1 & 3 & 3 & 2 & 2 & 4 & 1 & 4 & 2 & 4 & 1 \\
\hline $\mathbf{9 .}$ & 4 & 2 & 3 & 1 & 3 & 2 & 0 & 0 & 0 & 1 & 3 & 1 \\
\hline $\mathbf{1 0}$ & 4 & 2 & 4 & 2 & 1 & 3 & 2 & 1 & 1 & 2 & 4 & 2 \\
\hline 11. & 4 & 2 & 3 & 4 & 2 & 2 & 0 & 0 & 0 & 4 & 4 & 1 \\
\hline $\mathbf{1 2}$ & 4 & 2 & 4 & 4 & 2 & 3 & 2 & 1 & 1 & 4 & 4 & 2 \\
\hline
\end{tabular}

The coverage can be verified by, first, identifying the most similar characteristics of the sequence vectors belonging to the Near Misses cluster with the Pareto set scenarios $\overline{\boldsymbol{x}}$, and, then, by solving a Set Covering Problem (SCP) [Beasley et al., 1996; Di Maio et al., 2014c].

The most similar characteristics can be computed by Coverage vectors (one for each scenario belonging to $\overline{\boldsymbol{x}}$ ): this entails calculating the Hamming distance [Hamming, 1950] between each sequence vectors in $\overline{\boldsymbol{x}}$ and each one of the other sequence vectors in the Near Misses cluster [Popa et al., 2010]. The entries of the coverage vector (in our case twelve entries, one for time, magnitude and order of occurrence of each component failure, see Fig. 5) are increased if the Hamming distance between one same entry of the considered scenario belonging to $\overline{\boldsymbol{x}}$ and of the Near Misses vectors is equal to zero, as shown, without loss of generality, in Fig. 15 for 1 sequence vector of $\bar{x}$ and only 2 Near Misses vectors. 


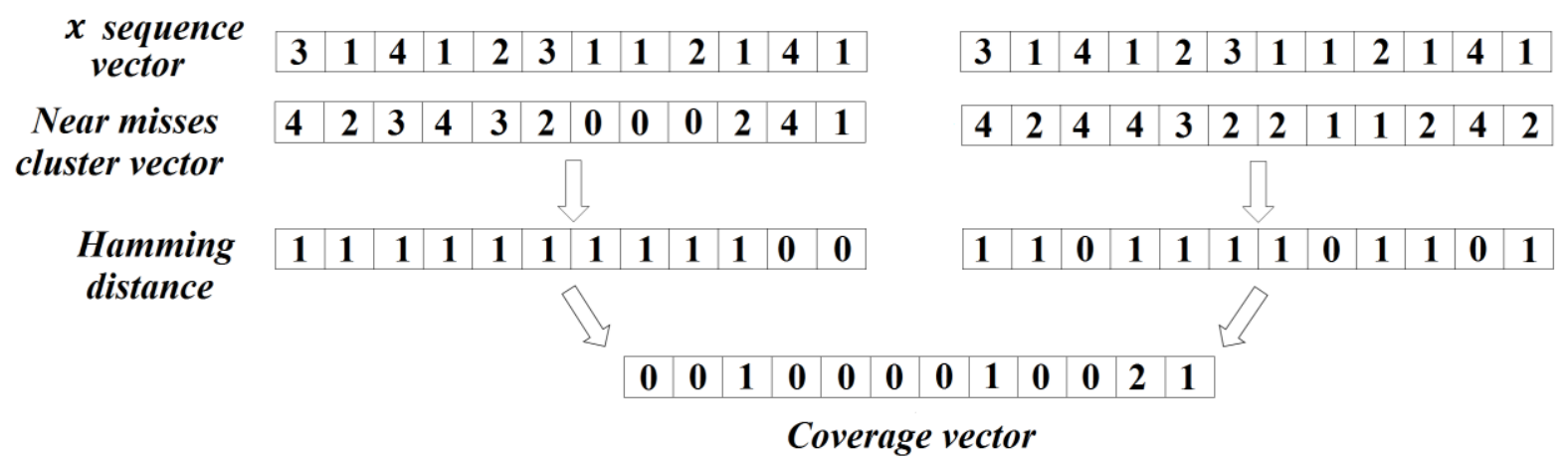

$x$ sequence

vector

Near misses

cluster vector

Hamming

distance

Fig. 15. Coverage vector computation by Hamming distance.

Table 4 lists the 12 coverage vectors, where each entry is the percentage of Near Misses vectors having the same stochastic behavior of the optimal set $\overline{\boldsymbol{x}}$ shown in Table 3. It can be seen that, for each scenario belonging to $\overline{\boldsymbol{x}}$, columns $8,11,12$ (e.g., Sensor-PID communication failure magnitude, PID failure magnitude and PID order of failure, respectively) have the largest values of the coverage vectors: this means that the majority of the sequence vectors of the Near Misses clusters can be well represented by (only) these failures. Furthermore, the analysis of the MVL values of the scenarios belonging to $\overline{\boldsymbol{x}}$ (Table 3 ) where the largest coverage values of these colums are registered (i.e, $87 \%$, $98.5 \%$ and $85.2 \%$ for columns 8,11 and 12, respectively) highlights that these failures are characterized by the same MVL values, that can be summarized as:

- the failure of the communication between the sensor monitoring the $N_{r l}$ and the PID controller;

- the failure of the PID controller with magnitude belonging to $[-5,-1] \%$ of the $Q_{e}$ value that should be provided at $P_{o}$, i.e., magnitude equal to 4 in MVL framework, and it is the first accident occurring along the sequence of events in over $85 \%$ of the Near Misses scenarios.

Table 4. List of coverage vectors for each scenario belonging to the Pareto set $\overline{\boldsymbol{x}}$.

\begin{tabular}{|c|c|c|c|c|c|c|c|c|c|c|c|c|}
\hline$\overline{\boldsymbol{x}}$ & $\begin{array}{c}\text { Steam } \\
\text { valve } \\
\text { failure } \\
\text { time }\end{array}$ & $\begin{array}{c}\text { Steam } \\
\text { valve } \\
\text { failure } \\
\text { magnitude }\end{array}$ & $\begin{array}{c}\text { Steam } \\
\text { valve } \\
\text { failure } \\
\text { order }\end{array}$ & $\begin{array}{c}\text { Safety } \\
\text { valve } \\
\text { failure } \\
\text { time }\end{array}$ & $\begin{array}{c}\text { Safety } \\
\text { valve } \\
\text { failure } \\
\text { magnitude }\end{array}$ & $\begin{array}{c}\text { Safety } \\
\text { valve } \\
\text { failure } \\
\text { order }\end{array}$ & $\begin{array}{c}\text { Sensor- } \\
\text { PID } \\
\text { failure } \\
\text { time }\end{array}$ & $\begin{array}{c}\text { Sensor- } \\
\text { PID } \\
\text { failure } \\
\text { magnitude }\end{array}$ & $\begin{array}{c}\text { Sensor- } \\
\text { PID } \\
\text { failure } \\
\text { order }\end{array}$ & $\begin{array}{c}\text { PID } \\
\text { failure } \\
\text { time }\end{array}$ & $\begin{array}{c}\text { PID } \\
\text { failure } \\
\text { magnitude }\end{array}$ & $\begin{array}{c}\text { PID } \\
\text { failure } \\
\text { order }\end{array}$ \\
\hline $\mathbf{1 .}$ & 11.4 & 11.4 & 11.4 & 8.1 & 30.4 & 0.6 & 25.3 & $\mathbf{8 7}$ & 46.4 & 1.2 & 1.5 & 0.6 \\
\hline $\mathbf{2 .}$ & 30.1 & 44.3 & 27.1 & 1.2 & 19.9 & 38 & 19.6 & $\mathbf{8 7}$ & 46.4 & 68.1 & $\mathbf{9 8 . 5}$ & $\mathbf{8 5 . 2}$ \\
\hline $\mathbf{3 .}$ & 30.1 & 44.3 & 27.1 & 8.1 & 19.9 & 38 & 22.3 & $\mathbf{8 7}$ & 14.2 & 27.4 & $\mathbf{9 8 . 5}$ & 14.2 \\
\hline $\mathbf{4 .}$ & 30.1 & 44.3 & 42.2 & 8.1 & 19.9 & 19.9 & 25.3 & $\mathbf{8 7}$ & 12.7 & 68.1 & $\mathbf{9 8 . 5}$ & $\mathbf{8 5 . 2}$ \\
\hline $\mathbf{5 .}$ & 30.1 & 44.3 & 27.1 & 28 & 30.4 & 38 & 22.3 & $\mathbf{8 7}$ & 46.4 & 68.1 & $\mathbf{9 8 . 5}$ & $\mathbf{8 5 . 2}$ \\
\hline $\mathbf{6 .}$ & 30.1 & 36.1 & 27.1 & 1.2 & 19.9 & 19.9 & 19.6 & $\mathbf{8 7}$ & 13.9 & 68.1 & $\mathbf{9 8 . 5}$ & $\mathbf{8 5 . 2}$ \\
\hline
\end{tabular}




\begin{tabular}{|c|c|c|c|c|c|c|c|c|c|c|c|c|}
\hline $\mathbf{7 .}$ & 30.1 & 36.1 & 27.1 & 8.1 & 30.4 & 0.6 & 22.3 & $\mathbf{8 7}$ & 46.4 & 3.3 & 1.5 & 0.60 \\
\hline $\mathbf{8 .}$ & 52.4 & 44.3 & 42.2 & 28 & 19.9 & 19.9 & 25.3 & $\mathbf{8 7}$ & 12.7 & 27.4 & $\mathbf{9 8 . 5}$ & $\mathbf{8 5 . 2}$ \\
\hline $\mathbf{9 .}$ & 52.4 & 36.1 & 42.2 & 1.2 & 30.4 & 19.9 & 13 & 13 & 13 & 68.1 & 1.5 & $\mathbf{8 5 . 2}$ \\
\hline $\mathbf{1 0 .}$ & 52.4 & 36.1 & 27.1 & 8.1 & 9.04 & 38 & 22.3 & $\mathbf{8 7}$ & 14.2 & 27.4 & $\mathbf{9 8 . 5}$ & 14.2 \\
\hline $\mathbf{1 1 .}$ & 52.4 & 36.1 & 42.2 & 51.2 & 19.9 & 19.9 & 13 & 13 & 13 & 1.2 & $\mathbf{9 8 . 5}$ & $\mathbf{8 5 . 2}$ \\
\hline $\mathbf{1 2 .}$ & 52.4 & 36.1 & 27.1 & 51.2 & 19.9 & 38 & 22.3 & $\mathbf{8 7}$ & 14.2 & 1.2 & $\mathbf{9 8 . 5}$ & 14.2 \\
\hline
\end{tabular}

A SCP can, thus, be solved for verifying that these latest characteristics are the minimum set of stochastic event occurrences and deterministic process variables values of $\overline{\boldsymbol{x}}$ that exhaustively describe the scenarios belonging to the Near Misses cluster: if a Near Miss sequence vector is characterized by (at least) one of the common characteristics, this is covered by the optimal set $\overline{\boldsymbol{x}}$. In the present application we have verified that all the scenarios belonging to the identified Near Misses cluster are covered by the minimal conditions that lead the system into a quasi-fault state, i.e., the optimal set $\overline{\boldsymbol{x}}$. In conclusion, it is sufficient the occurrence of one of the common characteristics listed above to lead the system in endangered and insecure operational conditions.

\section{CONCLUSIONS}

In this paper, a risk-based clustering approach for Near Misses identification has been proposed. The approach includes a risk-based feature selection task, where by each safe scenario is described in terms of probability, consequence and overall risk. The optimal features set is identified by a wrapper approach based on the combination of a MBDE algorithm with K-means clustering. The characteristics of the Near Misses scenarios are, then, identified solving a multi-objective optimization problem and Hamming distance as a measure of similarity.

The application of the approach to a case study of IDPSA of a UTSG has shown the possibility of retrieving relevant information for risk monitoring. 


\section{References}

[Aldemir et al., 2008] Aldemir, T., Guarro, S., Kirschenbaum, J., Mandelli, D., Mangan, L.A., Bucci, P., Yau, M., Johnson, B., Elks, C., Ekici, E., Stovsky, M.P., Miller, D.W., Sun, X., Arndt, S.A., “ $A$ Benchmark Implementation of Two Dynamic Methodologies for the Reliability Modeling of Digital Instrumentation and Control Systems”. NUREG-CR Report Draft, 2008.

[Aldemir, 2013] Aldemir T., "A survey of dynamic methodologies for probabilistic safety assessment of nuclear power plant", Annals of Nuclear Energy, Volume 52, pp.113-124, 2013.

[Aubry et al., 2012] Aubry J. F., Babykina G., Barros A., Brinzei N., Deleuze G., De Saporta B., Dufour F., Langeron Y., Zhang H., "Project APPRODYN: APPROches de la fiabilité DYNamique pour modéliser des systèmes critiques", Technical report, collaboration CRAN, EDF R\&D, INRIACQFD, UTT-ICD, 2012.

[Baraldi et al., 2014] Baraldi, P, Cannarile, F., Di Maio, F., Zio, E., "Hierarchical k-nearest neighbours classification and binary differential evolution for fault diagnostics of automotive bearings operating under variable conditions", under review, Reliability Engineering and System Safety, 2014.

[Beasley et al., 1996] Beasley, J.E., Chu, P.C., A genetic algorithm for the set covering problem, European Journal of Operational Research, vol.94, pp392-404, 1996.

[Bezdek, 1981] Bezdek J.C., "Pattern Recognition with Fuzzy Objective Function Algorithms", Kluwer Academic Publishers, Norwell, MA, USA, 1981.

[Bier et al., 1995] Bier, V.M., Yi, W. "The performance of precursor-based estimators for rare event frequencies", Reliability Engineering and System Safety, 50 (3), pp. 241-251, 1995.

[Calinski et al., 1974] Calinski, T., and J. Harabasz., "A dendrite method for cluster analysis." Communications in Statistics. Vol. 3, No. 1, pp. 1-27, 1974.

[Deb et al., 2002] Deb, K., Pratap, A., Agarwal, S., Meyarivan, T., “A fast and elitist multiobjective genetic algorithm: NSGA-II", IEEE Transactions on Evolutionary Computation, 6 (2), pp. 182-197, 2002.

[Devooght et al., 1992] Devooght D., Smidts C., "Probabilistic reactor dynamics I: the theory of continuous event trees”, Nuclear Science and Engineering, Volume 111, pp.229-240, 1992.

[Di Maio et al., 2013] Di Maio F., Baronchelli S., Vagnoli, M., Zio E., "Prime Implicants Determination by Differential Evolution for Dynamic Reliability Analysis of Non-Coherent Systems", under review, Reliability Engineering and System Safety.

[Di Maio et al., 2014a] F. Di Maio, S. Baronchelli, E. Zio, “A Computational framework for Prime Implicants Identification in non-coherent Dynamic Systems", Risk Analysis, 2014. 
[Di Maio et al., 2014b] Di Maio F., Baronchelli S., Zio E., “A visual interactive method for prime implicants identification”, IEEE Transactions on Reliability, 2014.

[Di Maio et al., 2014c] Di Maio, F., Baronchelli, S., Zio, E., Hierarchical Differential Evolution for Minimal Cut Sets Identification: Application to Nuclear Safety Systems, European Journal of Operational Research, Volume 238, Issue 2, Pages 645-652, 2014.

[Fukunaga et al., 1975] Fukunaga K., Hostetler L.D., "The estimation of the gradient of a density function, with application in pattern recognition", IEEE Transactions on Information Theory, IT-21 (1), pp. 32-40, 1975.

[Garibba et al., 1985] Garibba S., Guagnini E., Mussio P., "Multiple-Valued Logic Trees: Meaning and Prime Implicants”, IEEE Transactions on Reliability, Volume R-34, No. 5, pp.463-472, 1985 [Habibiyan et al., 2004] Habibiyan, H., Setayeshi, S., Arab-Alibeik, H. “A fuzzy-gain-scheduled neural controller for nuclear steam generators", Annals of Nuclear Energy, 31 (15), pp. 1765-1781, 2004.

[Hakobyan et al., 2008] Hakobyan A., Denning R., Aldemir T., Dunagan S., Kunsman D., “A Methodology for Generating Dynamic Accident Progression Event Trees for Level 2 PRA". SAND2008-4746, Sandia National Laboratories Albuquerque, New Mexico, 2008.

[Hamming, 1950] Hamming, R.W., "Error detecting and error correcting codes", Bell Syst. Tech. J.2, 147-160, 1950 .

[Hofer et al., 2004] Hofer E., Kloos M., Krzykacz-Hausmann B., Peschke J., Sonnenkalb M., “Dynamic Event Trees for Probabilistic Safety Analysis”, GRS, Garsching, Germany, 2004.

[Kang et al, 2013] Kang, D.G., Ahn, S.-H., Chang, S.H., "A combined deterministic and probabilistic procedure for safety assessment of beyond design basis accidents in nuclear power plant: Application to ECCS performance assessment for design basis LOCA redefinition", Nuclear Engineering and Design, 260, pp. 165-174, 2013.

[Keller et al., 2005] Keller, W., Modarres, M., “A historical overview of probabilistic risk assessment development and its use in the nuclear power industry: A tribute to the late Professor Norman Carl Rasmussen”, Reliability Engineering and System Safety, 89 (3), pp. 271-285, 2005.

[Khakzad et al., 2012] Khakzad, N., Khan, F., Amyotte, P. "Dynamic risk analysis using bow-tie approach”, Reliability Engineering and System Safety, 104, pp. 36-44, 2012.

[Kirschenbaum et al., 2009] Kirschenbaum J., Bucci P., Stovsky M., Mandelli D., Aldemir T., Yau M., Guarro S., Ekici E., Arndt S.A., "A benchmark system for comparing reliability modeling approaches for digital instrumentation and control systems", Nuclear Technology, Volume 165, pp.53-95, 2009. 
[Kohavi et al., 1997] Kohavi, R., John, G.H., "Wrappers for feature subset selection”, Artificial Intelligence, 97 (1-2), pp. 273-324, 1997.

[Kopustinskas et al., 2005] Kopustinskas V., Augutis J., Rimkevicius S., "Dynamic reliability and risk assessment of the accident localization system of the Ignalina NPP RBMK-1500 reactor", Reliability Engineering and System Safety, Volume 87, pp.77-87, 2005.

[Kothare et al., 2000] Kothare, M.V., Mettler, B., Morari, M., Bendotti, P., Falinower, C.-M., “Level control in the steam generator of a nuclear power plant", IEEE Transactions on Control Systems Technology, 8 (1), pp. 55-69, 2000.

[Kudinov et al., 2011] Vorobyev, Y., Kudinov, P., "Development and application of a genetic algorithm based dynamic pra methodology to plant vulnerability search", International Topical Meeting on Probabilistic Safety Assessment and Analysis 2011, 1, pp. 559-573, 2011.

[Johnson et al., 1996] Johnson, J.W., Rasmuson, D.M., “The US NRC's accident sequence precursor program: An overview and development of a Bayesian approach to estimate core damage frequency using precursor information”, Reliability Engineering and System Safety, 53 (2), pp. 205-216, 1996. [IAEA-TECDOC-981, 1997] “Assessment and management of ageing of major nuclear power plant component important to safety: Steam”, IAEA, IAEA-TECDOC-98, Vienna, ISSN 1011-4289, 1997. [Labeau et al., 2000] Labeau P.E., Smidts C., Swaminathan S., "Dynamic reliability: towards an integrated platform for probabilistic risk assessment”, Reliability Engineering and System Safety, Volume 68, pp.219-254, 2000.

[MacQueen, 1967] MacQueen J., "Some methods for classification and analysis of multivariate observations" Proceedings of 5th Berkeley Symposium on Mathematical Statistics and Probability, pages 281-297, 1967.

[Mandelli et al., 2010] Mandelli, D., Yilmaz, A., Metzroth, K., Aldemir, T., Denning, R., “Scenario aggregation and analysis via Mean-Shift Methodology", International Congress on Advances in Nuclear Power Plants 2010, ICAPP 2010, 2, pp. 987-991, 2010.

[Marseguerra et al., 1996] Marseguerra M., Zio E., "Monte Carlo approach to PSA for dynamic process system”, Reliability Engineering and System Safety, Volume 52, pp.227-241, 1996.

[Marseguerra et al., 2007] Marseguerra, M., Zio, E., Cadini, F., "Optimized adaptive fuzzy controller of the water level of a pressurized water reactor steam generator", Nuclear Science and Engineering, 155 (3), pp. 386-394, 2007.

[Popa et al., 2010] Popa, A., McDowell, J.J. The effect of Hamming distances in a computational model of selection by consequences”, Behavioural Processes, 84 (1), pp. 428-434, 2010.

[Quine, 1952] Quine W.V., "The problem of simplifying truth functions", Am. Math. Monthly, Volume 59, 521-531, 1952. 
[Rocco et al., 2004] Rocco C.M., Muselli M., “A Machine Learning Algorithm to Estimate Minimal Cut and Path Sets from a Monte Carlo Simulation", Proceedings Probabilistic Safety Assessment and Management PSAM7/ESREL'04, 2008.

[Saleh et al., 2013] Saleh, J.H., Saltmarsh, E.A., Favarò, F.M., Brevault, L., “Accident precursors, near misses, and warning signs: Critical review and formal definitions within the framework of Discrete Event Systems", Reliability Engineering and System Safety, 114 (1), pp. 148-154, 2013 [Zadakbar et al., 2013] Zadakbar, O., Imtiaz, S., Khan, F., "Dynamic risk assessment and fault detection using a multivariate technique”, Process Safety Progress, 32 (4), pp. 365-375, 2013.

[Zio et al., 2009] Zio E., Di Maio F., "Processing Dynamic Scenarios from a Reliability Analysis of a Nuclear Power Plant Digital Instrumentation and Control System", Annals of Nuclear Energy, Volume 36, pp.1386-1399, 2009.

[Zio et al., 2010] E. Zio, F. Di Maio, J. Tong, "Safety Margins Confidence Estimation for a Passive Residual Heat Removal System”, Reliability Engineering and System Safety, RESS, Vol. 95, 2010, pp. 828-836, doi:10.1016/j.ress.2010.03.006, 2010.

[Zio et al., 2012a] Zio, E.,Di Maio, F., "The needs and dreams for methodologies of IDPSA", Proceedings of the Integrated Deterministic and Probabilistic Safety Analyses workshop 20 November, KTH, Stockholm, Sweden, 2012.

[Zio et al., 2012b] Zio, E., Di Maio, F., Hu, J., Tse, P., Pecht, M., Tsui, K., “Ensemble-approaches for clustering health status of oil sand pumps", Expert Systems with Applications, 39 (5), pp. 4847 4859, 2012.

[Zio, 2014] Zio, E., "Integrated deterministic and probabilistic safety assessment: Concepts, challenges, research directions", Nuclear Engineering and Design, DOI: 10.1016/j.nucengdes.2014.09.004, 2014.

[Wang et al., 2010] Wang L., Fu X., Menhas M.I., “A Modified Binary Differential Evolution Algorithm", Life Modelling and Intelligent Computing, Lecture Notes in Computer Science, Volume 6329/2010, 2010.

\section{APPENDIX 1}

For a static reliability analysis of the UTSG, we conservatively assume that component failures occur at the beginning of the scenario, with magnitudes equal to their extreme (either maximum or minimum) plausible values [Zio et al., 2009]. We analyze the dynamic response of the system at constant $P_{o}$ values $\left(P_{o}=5 \% P_{n}\right.$ and $\left.P_{o}=80 \% P_{n}\right)$ and identify the Minimal Cuts Sets (MCS) with respect to the low and high level failure modes. Considering the binary, safe or faulty, states of the 6 
components, (component state is 0 if it works and 1 if it is failed), the number of possible system configurations is equal to $2^{6}$. However, many configurations are not detectable in constant power scenarios, e.g., simultaneous occurrence of low and high power communication failures, whereas some others are not important when event occurrence timing is not considered, e.g., PID and communication failures occur simultaneously, because, in this case, the feedback control output would always be the same as a stand-alone PID failure. Thus, the possible system configurations to be considered in a static analysis with constant power is equal to 12 for each power level (Table A.1).

Table A.1. Possible system configurations to be considered in the static reliability analysis with constant power profile

\begin{tabular}{|c|c|c|c|c|}
\hline $\begin{array}{c}\text { System } \\
\text { configurations }\end{array}$ & $\begin{array}{c}\text { Failure of the } \\
\text { Outlet Steam } \\
\text { Valve }\end{array}$ & $\begin{array}{c}\text { Failure of the } \\
\text { Safety Relief } \\
\text { Valve }\end{array}$ & $\begin{array}{c}\text { Level sensor- } \\
\text { PID controller } \\
\text { communication } \\
\text { interruption }\end{array}$ & $\begin{array}{c}\text { Failure of } \\
\text { the PID } \\
\text { controller }\end{array}$ \\
\hline 1 & - & - & - & - \\
\hline 2 & $\mathbf{X}$ & - & - & - \\
\hline 3 & - & $\mathbf{X}$ & - & - \\
\hline 4 & - & - & $\mathbf{X}$ & - \\
\hline 5 & - & - & - & $\mathbf{X}$ \\
\hline $\mathbf{6}$ & $\mathbf{X}$ & $\mathbf{X}$ & - & - \\
\hline 7 & $\mathbf{X}$ & - & $\mathbf{X}$ & - \\
\hline $\mathbf{8}$ & $\mathbf{X}$ & - & - & $\mathbf{X}$ \\
\hline $\mathbf{9}$ & - & $\mathbf{X}$ & $\mathbf{X}$ & - \\
\hline 10 & - & $\mathbf{X}$ & - & $\mathbf{X}$ \\
\hline 11 & $\mathbf{X}$ & $\mathbf{X}$ & $\mathbf{X}$ & - \\
\hline 12 & $\mathbf{X}$ & $\mathbf{X}$ & - & $\mathbf{X}$ \\
\hline
\end{tabular}

To identify the system MCS, the different system configurations of Table A.1 have been simulated by the SIMULINK model, at low and at high (constant) power levels. It turns out that the MCSs for the high level failure mode are the same at both power levels (Fig. A.1): the failure of the PID controller at its minimum values (i.e., $-18 \%$ of the nominal $Q_{e}$ that should be provided at $P_{o}$ ) and of the steam valve at its maximum value (i.e., $150 \%$ of the nominal $Q_{v}$ value that should be provided at $P_{o}$ ) are two first order MCS. The $N_{r l}$ evolutions when these MCSs occur are shown in Figs. A.2 and A.3. 


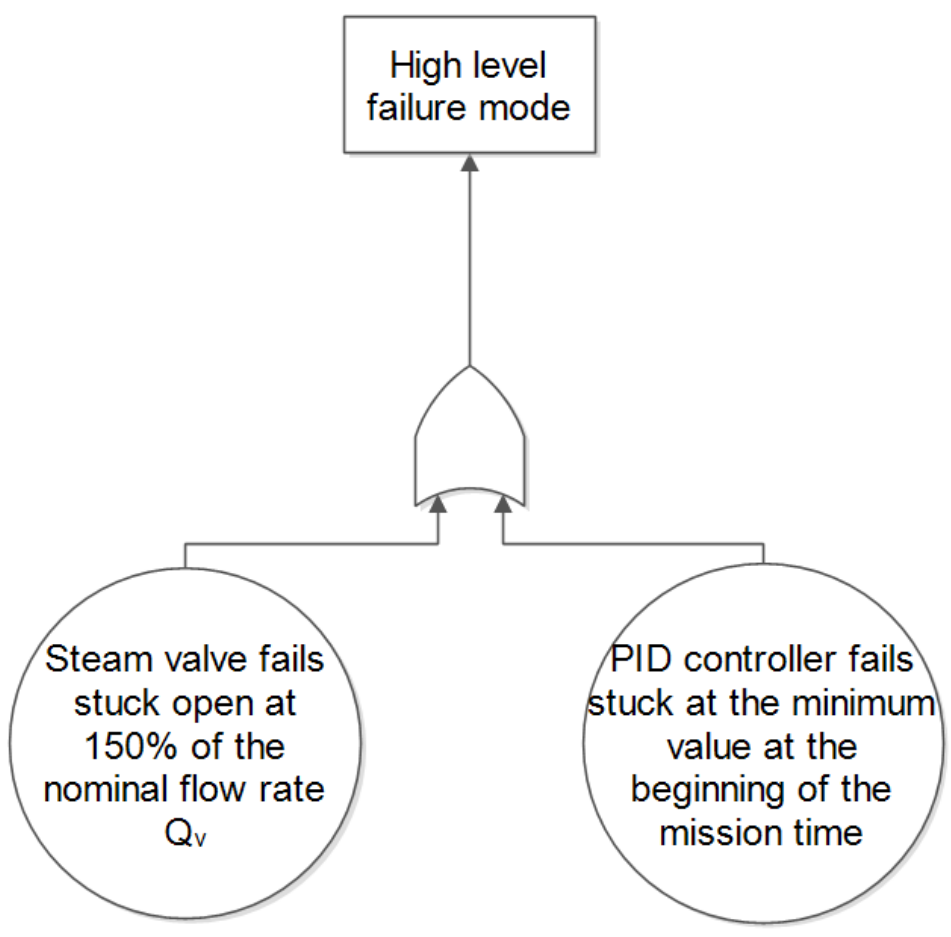

Fig. A.1. Fault Tree for the high level failure mode

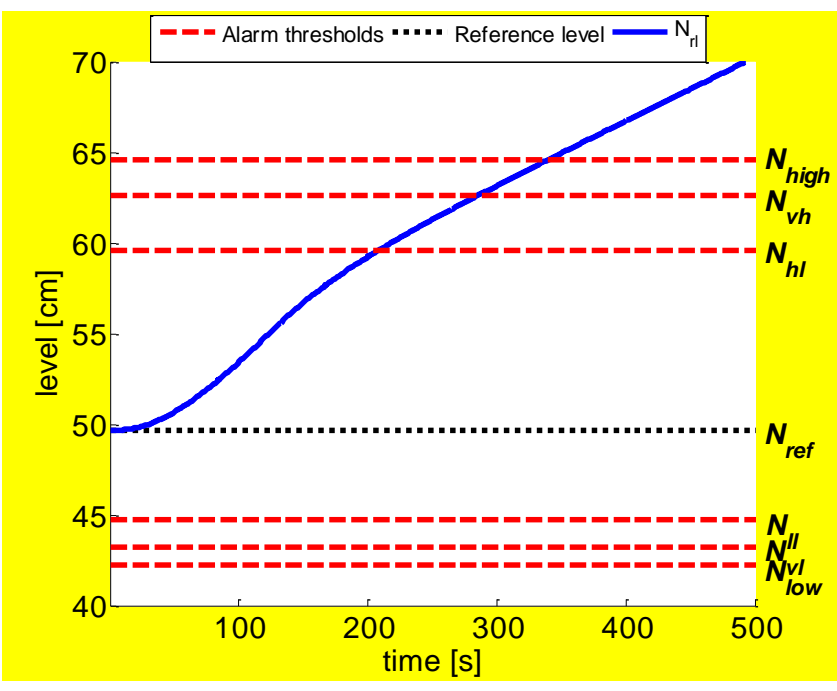

(a)

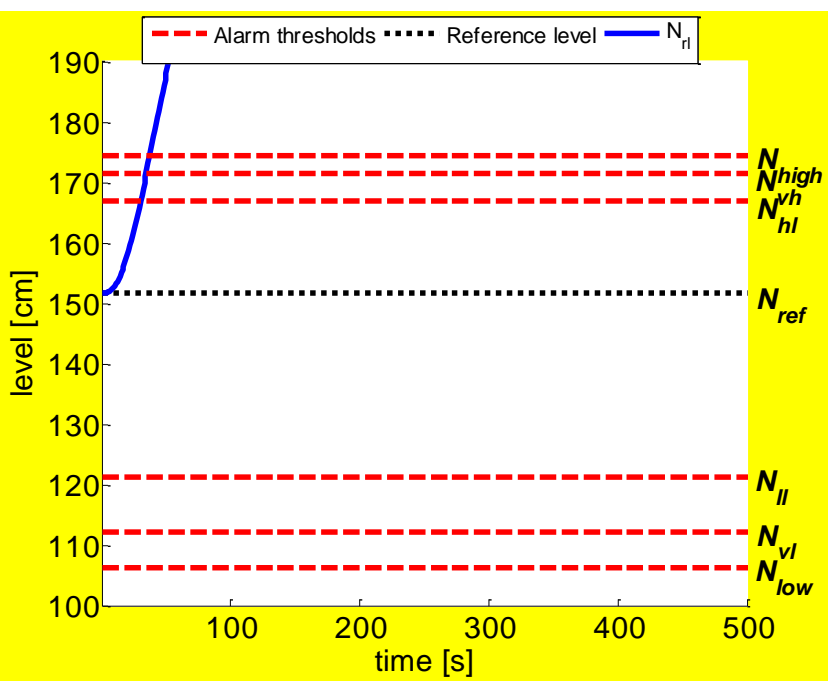

(b)

Fig. A.2. $N_{r l}$ evolution when the PID controller output is stuck at time $t=0$ at the minimum allowable value of $-18 \%$ of nominal $Q_{e}$ that should be provided at $5 \% P_{n}(a)$ and at $80 \% P_{n}(b)$. 


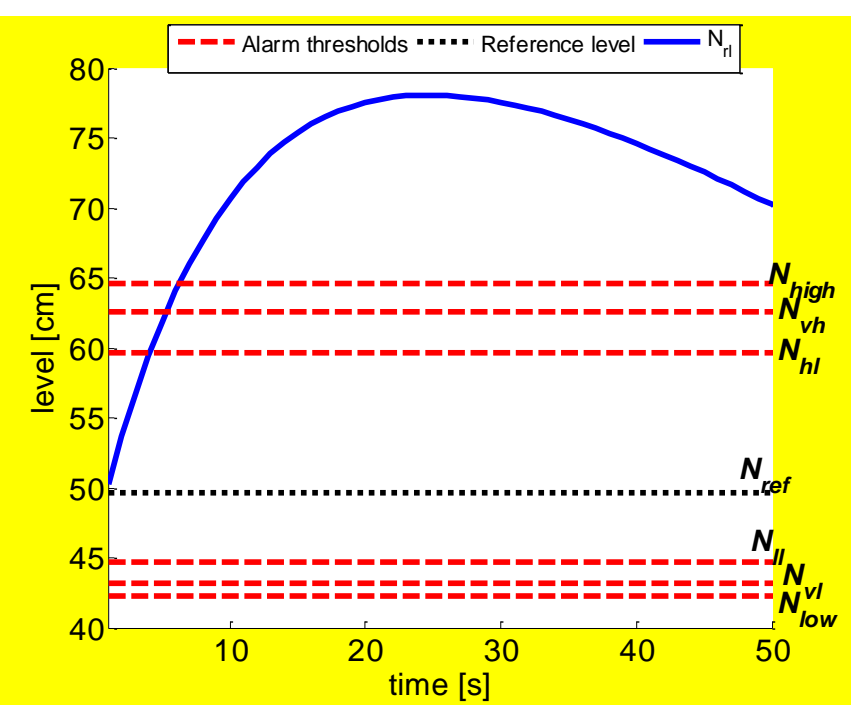

(b)

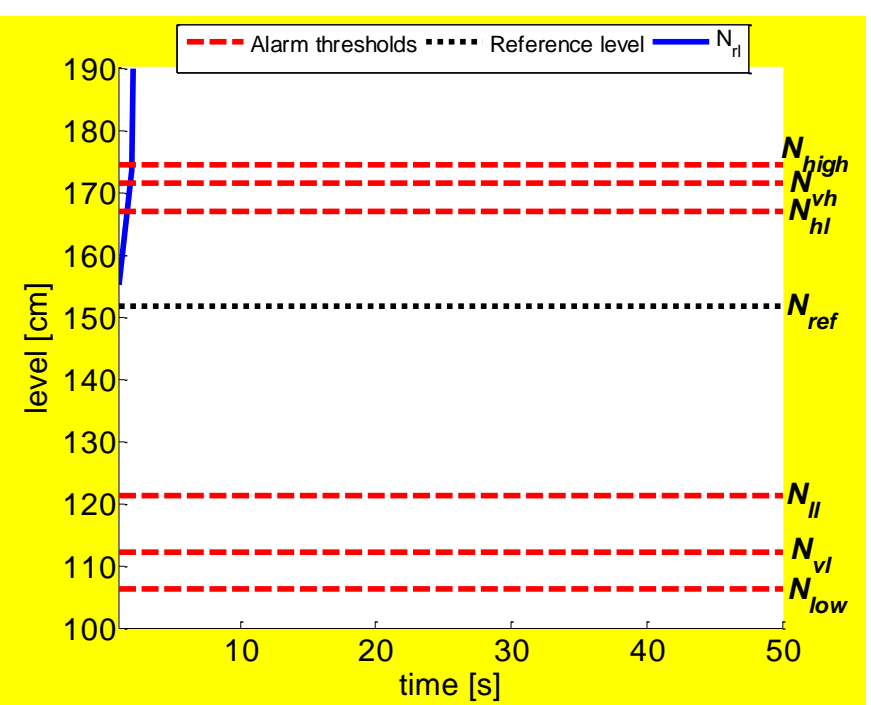

(b)

Fig. A.3. $N_{r l}$ evolution when the steam valve fails stuck at time $t=0$ at the maximum allowable value of $150 \%$ of nominal $Q_{v}$ that should be provided at $5 \% P_{n}(a)$ and at $80 \% P_{n}(b)$.

The analysis of the low level failure mode provides different MCSs at different $P_{o}$. At $5 \% P_{n}$, there are three first order MCSs represented by: i) Safety valve fails stuck at the maximum allowable value, i.e., $Q_{s f}=50.5(\mathrm{~kg} / \mathrm{s})$; ii) Steam valve fails stuck closed; iii) PID controller fails stuck at its maximum values, (i.e., $18 \%$ of the nominal $Q_{e}$ value that should be provided at $5 \% P_{n}$ ). The $N_{r l}$ evolution when these MCSs occur and the relative FT are shown in Fig. A.4 and Fig. A.5, respectively.

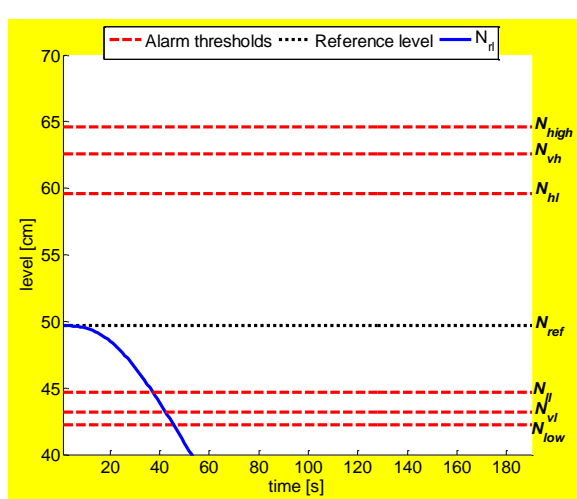

(a)

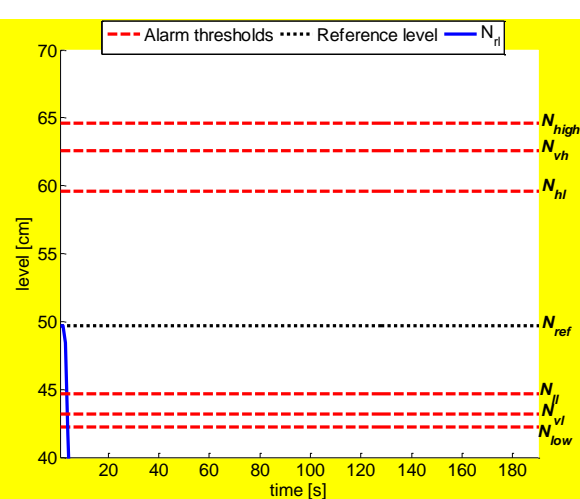

(b)

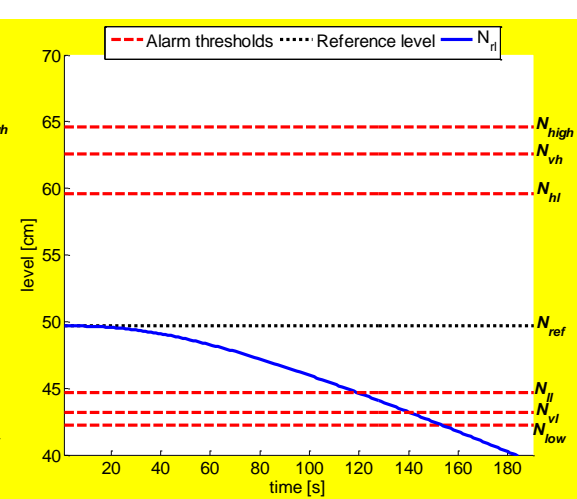

(c)

Fig. A.4. (a) $N_{r l}$ evolution when the safety relief valve fails stuck with $Q_{s f}=50.5(\mathrm{~kg} / \mathrm{s}) ;(\mathrm{b})$ the steam valve fails stuck closed; (c) the PID controller fails stuck at $18 \%$ of nominal $Q_{e}$ that should be provided at $5 \% P_{n}$. 


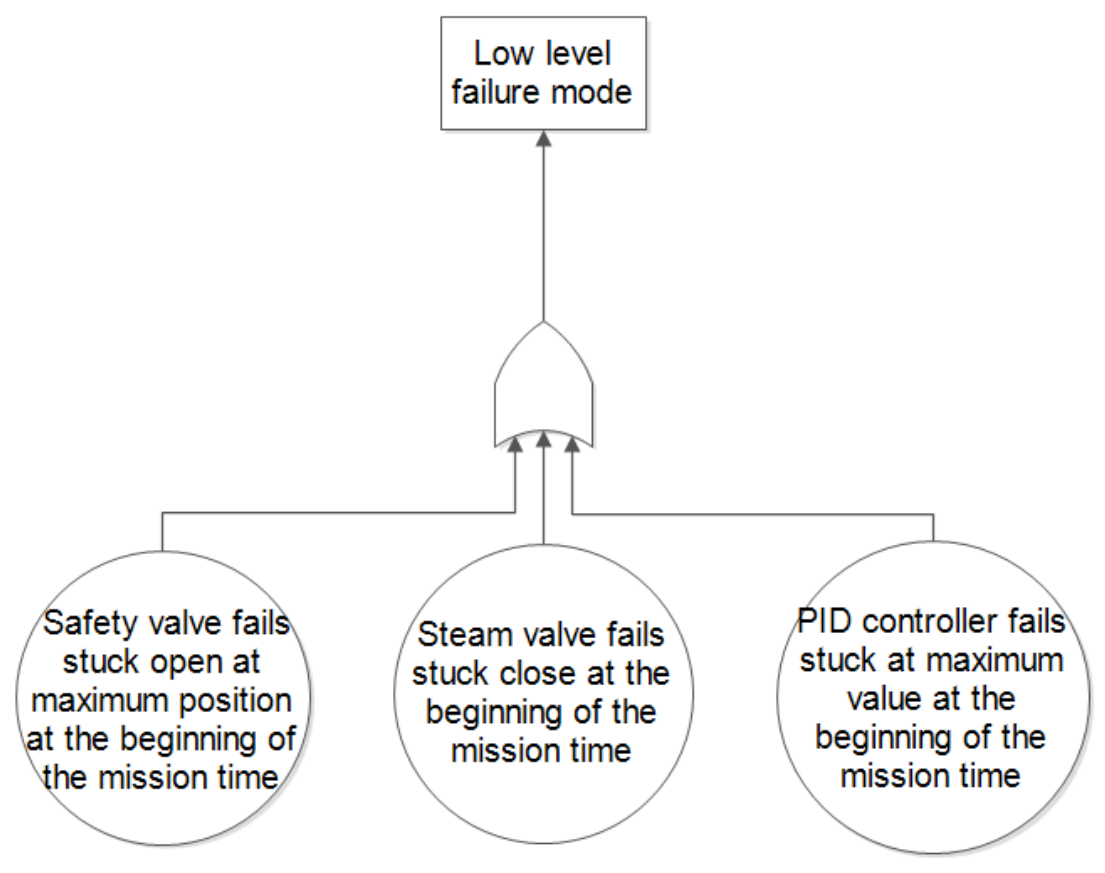

Fig. A.5. Fault Tree for the low level failure mode at low power.

At $80 \% P_{n}$, three MCSs are found: i) a second-order MCS that combines the failure of the safety relief valve at its maximum allowable value, i.e., $Q_{s f}=50.5(\mathrm{~kg} / \mathrm{s})$, and the failure of the communication, ii) the steam valve failure in a closed position and iii) the PID controller fails at its maximum value (i.e., $18 \%$ of the nominal $Q_{e}$ value that should be provided at $\left.80 \% P_{n}\right)$. The $N_{r l}$ evolution when these MCSs occur and the relative FT are shown in Fig. A.6 and Fig. A.7, respectively.
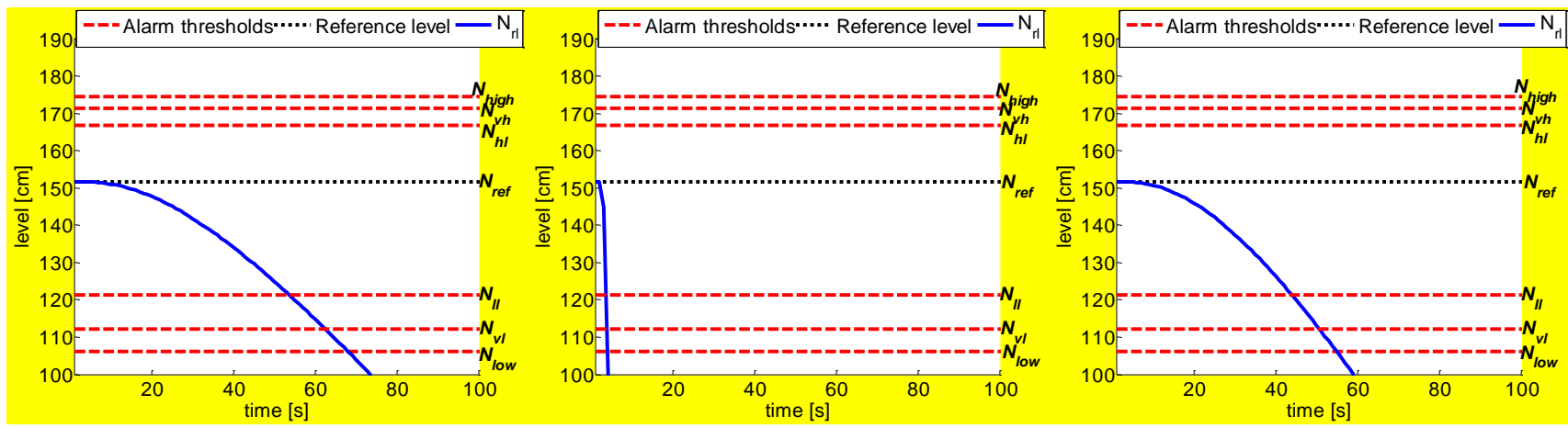

(a)

(b)

(c) 
Fig. A.6. (a) $N_{r l}$ evolution when simultaneously the safety relief valve fails stuck with $Q_{s f}=50.5$ $(\mathrm{kg} / \mathrm{s})$ and the communication fails; (b) the steam valve fails stuck closed; (c) the PID controller fails stuck at $18 \%$ of the nominal $Q_{e}$ that should be provided at $80 \% P_{n}$.

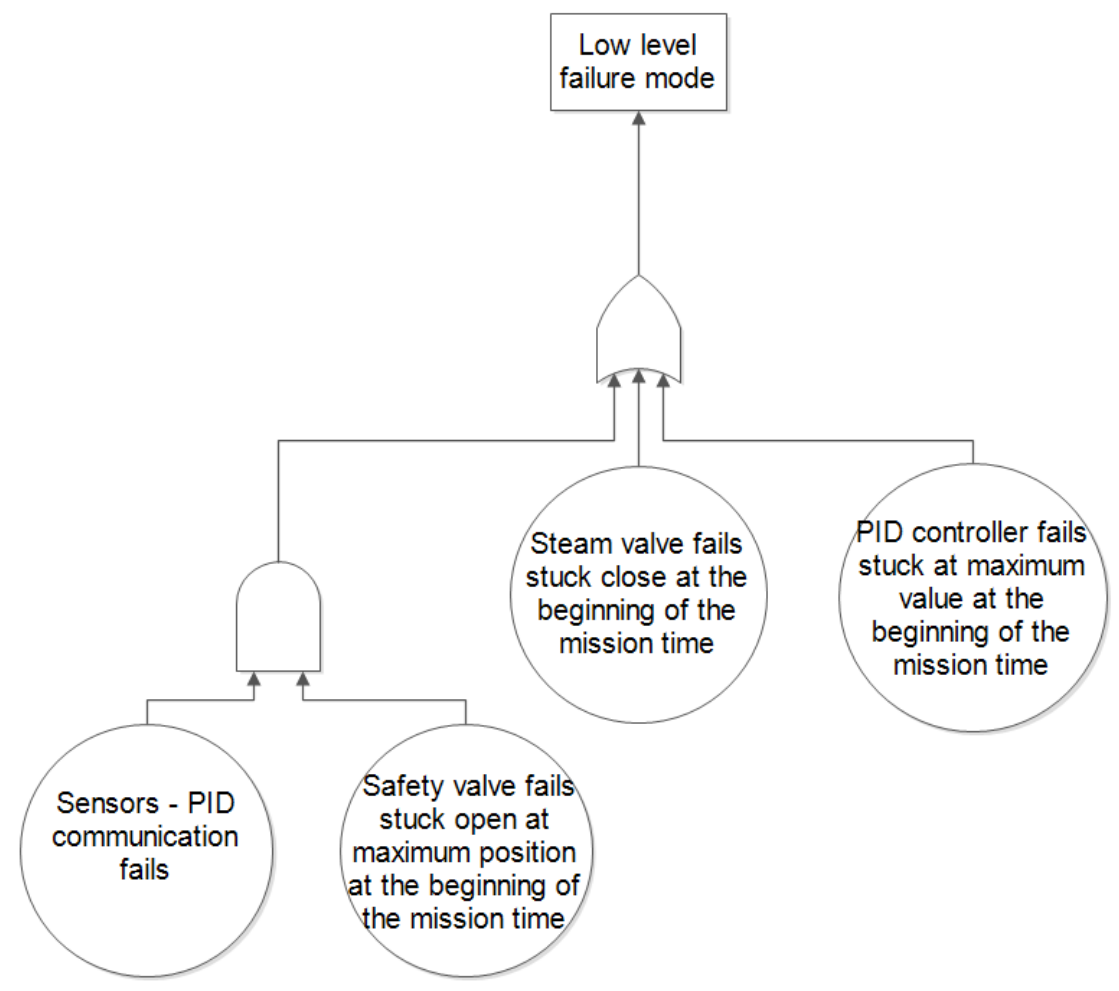

Fig. A.7. Fault Tree for the low level failure mode at high power

\section{APPENDIX 2}

\begin{tabular}{|c|c|c|c|c|c|c|c|c|c|c|c|c|c|c|c|c|c|c|c|c|c|c|c|c|c|}
\hline $\begin{array}{c}\text { Near } \\
\text { Miss }\end{array}$ & $T_{s}$ & $M_{s}$ & $O_{s}$ & $T_{s a}$ & $M_{s a}$ & $O_{s a}$ & $T_{c}$ & $M_{c}$ & $O_{c}$ & $T_{p}$ & $M_{p}$ & $O_{p}$ & $\begin{array}{c}\text { Near } \\
\text { Miss }\end{array}$ & $T_{s}$ & $M_{s}$ & $O_{s}$ & $T_{s a}$ & $M_{s a}$ & $O_{s a}$ & $T_{c}$ & $M_{c}$ & $O_{c}$ & $T_{p}$ & $M_{p}$ & $O_{p}$ \\
\hline 1. & 0 & 0 & 0 & 0 & 0 & 0 & 0 & 0 & 0 & 2 & 4 & 1 & 167 & 4 & 1 & 3 & 0 & 0 & 0 & 4 & 1 & 2 & 1 & 4 & 1 \\
\hline 2. & 0 & 0 & 0 & 0 & 0 & 0 & 1 & 1 & 1 & 1 & 4 & 2 & 168 & 4 & 1 & 3 & 0 & 0 & 0 & 4 & 1 & 2 & 2 & 4 & 1 \\
\hline 3. & 0 & 0 & 0 & 0 & 0 & 0 & 1 & 1 & 2 & 1 & 4 & 1 & 169 & 4 & 1 & 4 & 2 & 2 & 3 & 1 & 1 & 2 & 1 & 4 & 1 \\
\hline 4. & 0 & 0 & 0 & 0 & 0 & 0 & 2 & 1 & 1 & 2 & 4 & 2 & 170. & 4 & 1 & 4 & 2 & 2 & 2 & 3 & 1 & 3 & 2 & 4 & 1 \\
\hline 5. & 0 & 0 & 0 & 0 & 0 & 0 & 2 & 1 & 2 & 2 & 4 & 1 & 171. & 4 & 1 & 4 & 2 & 3 & 3 & 1 & 1 & 2 & 1 & 4 & 1 \\
\hline 6. & 0 & 0 & 0 & 0 & 0 & 0 & 2 & 1 & 1 & 3 & 4 & 2 & 172. & 4 & 1 & 4 & 2 & 4 & 2 & 4 & 1 & 3 & 1 & 4 & 1 \\
\hline 7. & 0 & 0 & 0 & 0 & 0 & 0 & 4 & 1 & 2 & 2 & 4 & 1 & 173. & 4 & 1 & 4 & 3 & 2 & 3 & 1 & 1 & 1 & 1 & 4 & 2 \\
\hline 8. & 0 & 0 & 0 & 2 & 2 & 2 & 4 & 1 & 3 & 2 & 4 & 1 & 174. & 4 & 1 & 3 & 3 & 2 & 2 & 4 & 1 & 4 & 2 & 4 & 1 \\
\hline 9. & 0 & 0 & 0 & 2 & 3 & 1 & 4 & 1 & 2 & 4 & 3 & 3 & 175. & 4 & 1 & 3 & 3 & 3 & 2 & 0 & 0 & 0 & 1 & 4 & 1 \\
\hline 10. & 0 & 0 & 0 & 2 & 4 & 2 & 4 & 1 & 3 & 1 & 4 & 1 & 176. & 4 & 1 & 4 & 3 & 3 & 3 & 1 & 1 & 2 & 1 & 4 & 1 \\
\hline 11. & 0 & 0 & 0 & 3 & 2 & 3 & 3 & 1 & 2 & 1 & 4 & 1 & 177. & 4 & 1 & 4 & 3 & 3 & 3 & 2 & 1 & 2 & 1 & 4 & 1 \\
\hline 12. & 0 & 0 & 0 & 3 & 3 & 3 & 1 & 1 & 1 & 1 & 4 & 2 & 178. & 4 & 1 & 3 & 3 & 4 & 2 & 0 & 0 & 0 & 1 & 4 & 1 \\
\hline 13. & 0 & 0 & 0 & 3 & 3 & 3 & 1 & 1 & 2 & 1 & 4 & 1 & 179 & 4 & 1 & 4 & 3 & 4 & 3 & 1 & 1 & 1 & 1 & 4 & 2 \\
\hline
\end{tabular}




\begin{tabular}{|c|c|c|c|c|c|c|c|c|c|c|c|c|c|c|c|c|c|c|c|c|c|c|c|c|c|}
\hline 14. & 0 & 0 & 0 & 3 & 3 & 3 & 2 & 1 & 2 & 1 & 4 & 1 & 180. & \begin{tabular}{l|l}
4 \\
\end{tabular} & 1 & 4 & 3 & 4 & 3 & 2 & 1 & 2 & 2 & 4 & 1 \\
\hline 15. & 0 & 0 & 0 & 3 & 3 & 3 & 2 & 1 & 2 & 2 & 4 & 1 & 181. & 4 & 1 & 4 & 3 & 4 & 3 & 3 & 1 & 2 & 1 & 4 & 1 \\
\hline 16. & 0 & 0 & 0 & 3 & 3 & 2 & 3 & 1 & 3 & 1 & 4 & 1 & 182. & 4 & 1 & 4 & 3 & 4 & 3 & 3 & 1 & 2 & 2 & 4 & 1 \\
\hline 17. & 0 & 0 & 0 & 3 & 3 & 2 & 4 & 1 & 3 & 1 & 4 & 1 & 183. & 4 & 1 & 3 & 3 & 4 & 2 & 4 & 1 & 4 & 2 & 4 & 1 \\
\hline 18. & 0 & 0 & 0 & 3 & 4 & 3 & 1 & 1 & 1 & 2 & 4 & 2 & 184. & 4 & 1 & 2 & 4 & 1 & 3 & 0 & 0 & 0 & 1 & 4 & 1 \\
\hline 19. & 0 & 0 & 0 & 3 & 4 & 3 & 3 & 1 & 2 & 1 & 4 & 1 & 185. & 4 & 1 & 2 & 4 & 1 & 3 & 0 & 0 & 0 & 2 & 4 & 1 \\
\hline 20. & 0 & 0 & 0 & 4 & 2 & 2 & 0 & 0 & 0 & 4 & 4 & 1 & 186. & 4 & 1 & 3 & 4 & 1 & 4 & 1 & 1 & 1 & 2 & 4 & 2 \\
\hline 21. & 0 & 0 & 0 & 4 & 2 & 3 & 2 & 1 & 2 & 1 & 4 & 1 & 187. & 4 & 1 & 3 & 4 & 1 & 4 & 2 & 1 & 2 & 1 & 4 & 1 \\
\hline 22. & 0 & 0 & 0 & 4 & 2 & 3 & 3 & 1 & 2 & 1 & 4 & 1 & 188. & 4 & 1 & 3 & 4 & 1 & 4 & 2 & 1 & 2 & 2 & 4 & 1 \\
\hline 23. & 0 & 0 & 0 & 4 & 2 & 3 & 4 & 1 & 2 & 1 & 4 & 1 & 189. & 4 & 1 & 3 & 4 & 1 & 4 & 3 & 1 & 2 & 1 & 4 & 1 \\
\hline 24. & 0 & 0 & 0 & 4 & 3 & 2 & 0 & 0 & 0 & 1 & 4 & 1 & 190. & 4 & 1 & 2 & 4 & 1 & 3 & 4 & 1 & 4 & 1 & 4 & 1 \\
\hline 25. & 0 & 0 & 0 & 4 & 3 & 2 & 0 & 0 & 0 & 2 & 4 & 1 & 191. & 4 & 1 & 3 & 4 & 1 & 4 & 4 & 1 & 2 & 1 & 4 & 1 \\
\hline 26. & 0 & 0 & 0 & 4 & 3 & 3 & 2 & 1 & 1 & 2 & 4 & 2 & 192. & 4 & 1 & 2 & 4 & 1 & 3 & 4 & 1 & 4 & 2 & 4 & 1 \\
\hline 27. & 0 & 0 & 0 & 4 & 3 & 3 & 3 & 1 & 2 & 2 & 4 & 1 & 193. & 4 & 1 & 3 & 4 & 1 & 4 & 4 & 1 & 2 & 3 & 4 & 1 \\
\hline 28. & 0 & 0 & 0 & 4 & 3 & 2 & 4 & 1 & 3 & 1 & 4 & 1 & 194. & 4 & 1 & 2 & 4 & 2 & 3 & 0 & 0 & 0 & 1 & 4 & 1 \\
\hline 29. & 0 & 0 & 0 & 4 & 4 & 2 & 0 & 0 & 0 & 1 & 4 & 1 & 195. & 4 & 1 & 3 & 4 & 2 & 4 & 1 & 1 & 1 & 1 & 4 & 2 \\
\hline 30. & 0 & 0 & 0 & 4 & 4 & 2 & 0 & 0 & 0 & 2 & 4 & 1 & 196. & 4 & 1 & 4 & 4 & 2 & 3 & 1 & 1 & 2 & 1 & 4 & 1 \\
\hline 31. & 0 & 0 & 0 & 4 & 4 & 3 & 1 & 1 & 1 & 1 & 4 & 2 & 197. & 4 & 1 & 3 & 4 & 2 & 4 & 2 & 1 & 2 & 1 & 4 & 1 \\
\hline 32. & 0 & 0 & 0 & 4 & 4 & 3 & 1 & 1 & 2 & 1 & 4 & 1 & 198. & 4 & 1 & 4 & 4 & 2 & 3 & 2 & 1 & 1 & 2 & 4 & 2 \\
\hline 33. & 0 & 0 & 0 & 4 & 4 & 3 & 1 & 1 & 1 & 2 & 4 & 2 & 199. & 4 & 1 & 3 & 4 & 2 & 4 & 2 & 1 & 2 & 2 & 4 & 1 \\
\hline 34. & 0 & 0 & 0 & 4 & 4 & 3 & 2 & 1 & 2 & 1 & 4 & 1 & 200. & 4 & 1 & 3 & 4 & 2 & 4 & 3 & 1 & 2 & 2 & 4 & 1 \\
\hline 35. & 0 & 0 & 0 & 4 & 4 & 3 & 2 & 1 & 1 & 2 & 4 & 2 & 201. & 4 & 1 & 2 & 4 & 2 & 3 & 4 & 1 & 4 & 1 & 4 & 1 \\
\hline 36. & 0 & 0 & 0 & 4 & 4 & 3 & 3 & 1 & 2 & 3 & 4 & 1 & 202. & 4 & 1 & 3 & 4 & 2 & 2 & 4 & 1 & 4 & 1 & 4 & 1 \\
\hline 37. & 0 & 0 & 0 & 4 & 4 & 3 & 4 & 1 & 2 & 1 & 4 & 1 & 203. & 4 & 1 & 3 & 4 & 2 & 4 & 4 & 1 & 2 & 1 & 4 & 1 \\
\hline 38. & 0 & 0 & 0 & 4 & 4 & 3 & 4 & 1 & 2 & 2 & 4 & 1 & 204. & 4 & 1 & 2 & 4 & 2 & 4 & 4 & 1 & 3 & 2 & 4 & 1 \\
\hline 39. & 1 & 1 & 3 & 1 & 2 & 2 & 1 & 1 & 4 & 1 & 4 & 1 & 205. & 4 & 1 & 3 & 4 & 2 & 4 & 4 & 1 & 2 & 2 & 4 & 1 \\
\hline 40. & 2 & 1 & 2 & 0 & 0 & 0 & 0 & 0 & 0 & 1 & 4 & 1 & 206. & 4 & 1 & 4 & 4 & 2 & 2 & 4 & 1 & 3 & 3 & 4 & 1 \\
\hline 41. & 2 & 1 & 3 & 0 & 0 & 0 & 1 & 1 & 2 & 1 & 4 & 1 & 207. & 4 & 1 & 2 & 4 & 3 & 3 & 0 & 0 & 0 & 1 & 4 & 1 \\
\hline 42. & 2 & 1 & 3 & 2 & 2 & 2 & 0 & 0 & 0 & 2 & 4 & 1 & 208 . & 4 & 1 & 3 & 4 & 3 & 2 & 0 & 0 & 0 & 1 & 4 & 1 \\
\hline 43. & 2 & 1 & 2 & 2 & 2 & 3 & 3 & 1 & 4 & 1 & 4 & 1 & 209. & 4 & 1 & 4 & 4 & 3 & 3 & 1 & 1 & 1 & 1 & 4 & 2 \\
\hline 44. & 2 & 1 & 4 & 2 & 4 & 2 & 2 & 1 & 3 & 1 & 3 & 1 & 210. & 4 & 1 & 3 & 4 & 3 & 4 & 1 & 1 & 2 & 1 & 4 & 1 \\
\hline 45. & 2 & 1 & 3 & 2 & 4 & 4 & 2 & 1 & 2 & 1 & 4 & 1 & 211. & 4 & 1 & 4 & 4 & 3 & 3 & 1 & 1 & 2 & 1 & 4 & 1 \\
\hline 46. & 2 & 1 & 3 & 3 & 2 & 4 & 2 & 1 & 2 & 1 & 4 & 1 & 212. & 4 & 1 & 3 & 4 & 3 & 4 & 2 & 1 & 2 & 1 & 4 & 1 \\
\hline 47. & 2 & 1 & 2 & 3 & 2 & 3 & 4 & 1 & 4 & 1 & 4 & 1 & 213. & 4 & 1 & 4 & 4 & 3 & 3 & 2 & 1 & 2 & 1 & 4 & 1 \\
\hline 48. & 2 & 1 & 3 & 3 & 3 & 4 & 2 & 1 & 2 & 1 & 4 & 1 & 214. & 4 & 1 & 4 & 4 & 3 & 3 & 2 & 1 & 1 & 2 & 4 & 2 \\
\hline 49. & 2 & 1 & 2 & 3 & 3 & 3 & 3 & 1 & 4 & 1 & 4 & 1 & 215. & 4 & 1 & 3 & 4 & 3 & 4 & 2 & 1 & 2 & 2 & 4 & 1 \\
\hline 50. & 2 & 1 & 3 & 3 & 4 & 4 & 1 & 1 & 2 & 1 & 4 & 1 & 216. & 4 & 1 & 4 & 4 & 3 & 3 & 2 & 1 & 2 & 2 & 4 & 1 \\
\hline 51. & 2 & 1 & 2 & 3 & 4 & 3 & 3 & 1 & 4 & 1 & 4 & 1 & 217. & 4 & 1 & 3 & 4 & 3 & 4 & 3 & 1 & 2 & 1 & 4 & 1 \\
\hline 52. & 2 & 1 & 3 & 4 & 1 & 4 & 2 & 1 & 2 & 1 & 4 & 1 & 218. & 4 & 1 & 3 & 4 & 3 & 4 & 3 & 1 & 2 & 3 & 4 & 1 \\
\hline 53. & 2 & 1 & 3 & 4 & 3 & 4 & 1 & 1 & 2 & 1 & 4 & 1 & 219. & 4 & 1 & 2 & 4 & 3 & 3 & 4 & 1 & 4 & 1 & 4 & 1 \\
\hline 54. & 2 & 1 & 2 & 4 & 3 & 4 & 3 & 1 & 3 & 1 & 4 & 1 & 220. & 4 & 1 & 2 & 4 & 3 & 4 & 4 & 1 & 3 & 1 & 4 & 1 \\
\hline 55. & 2 & 1 & 2 & 4 & 3 & 4 & 4 & 1 & 3 & 1 & 4 & 1 & 221. & 4 & 1 & 3 & 4 & 3 & 2 & 4 & 1 & 4 & 1 & 4 & 1 \\
\hline 56. & 2 & 1 & 2 & 4 & 4 & 3 & 0 & 0 & 0 & 1 & 4 & 1 & 222. & 4 & 1 & 3 & 4 & 3 & 4 & 4 & 1 & 2 & 1 & 4 & 1 \\
\hline 57. & 2 & 2 & 3 & 3 & 4 & 4 & 1 & 1 & 2 & 1 & 4 & 1 & 223. & 4 & 1 & 2 & 4 & 3 & 3 & 4 & 1 & 4 & 2 & 4 & 1 \\
\hline 58. & 2 & 2 & 3 & 4 & 4 & 4 & 2 & 1 & 2 & 1 & 4 & 1 & 224. & 4 & 1 & 3 & 4 & 3 & 4 & 4 & 1 & 2 & 2 & 4 & 1 \\
\hline 59. & 3 & 1 & 2 & 0 & 0 & 0 & 0 & 0 & 0 & 1 & 4 & 1 & 225. & 4 & 1 & 4 & 4 & 3 & 2 & 4 & 1 & 3 & 2 & 4 & 1 \\
\hline 60. & 3 & 1 & 3 & 0 & 0 & 0 & 1 & 1 & 1 & 1 & 4 & 2 & 226. & 4 & 1 & 2 & 4 & 4 & 3 & 0 & 0 & 0 & 2 & 4 & 1 \\
\hline 61. & 3 & 1 & 3 & 0 & 0 & 0 & 1 & 1 & 2 & 1 & 4 & 1 & 227. & 4 & 1 & 3 & 4 & 4 & 4 & 1 & 1 & 1 & 1 & 4 & 2 \\
\hline 62. & 3 & 1 & 3 & 0 & 0 & 0 & 2 & 1 & 2 & 1 & 3 & 1 & 228. & 4 & 1 & 4 & 4 & 4 & 3 & 1 & 1 & 1 & 1 & 4 & 2 \\
\hline
\end{tabular}




\begin{tabular}{|c|c|c|c|c|c|c|c|c|c|c|c|c|c|c|c|c|c|c|c|c|c|c|c|c|c|}
\hline 63. & 3 & 1 & 3 & 0 & 0 & 0 & 2 & 1 & 2 & 1 & 4 & 1 & 229. & 4 & 1 & 4 & 4 & 4 & 3 & 1 & 1 & 1 & 2 & 4 & 2 \\
\hline 64. & 3 & 1 & 3 & 0 & 0 & 0 & 2 & 1 & 2 & 2 & 4 & 1 & 230 & 4 & 1 & 3 & 4 & 4 & 4 & 2 & 1 & 2 & 1 & 4 & 1 \\
\hline 65. & 3 & 1 & 4 & 1 & 2 & 3 & 1 & 1 & 2 & 1 & 4 & 1 & 231. & 4 & 1 & 4 & 4 & 4 & 3 & 2 & 1 & 2 & 1 & 4 & 1 \\
\hline 66. & 3 & 1 & 4 & 2 & 2 & 2 & 2 & 1 & 3 & 2 & 4 & 1 & 232. & 4 & 1 & 4 & 4 & 4 & 3 & 2 & 1 & 2 & 2 & 4 & 1 \\
\hline 67. & 3 & 1 & 4 & 2 & 2 & 3 & 2 & 1 & 1 & 2 & 4 & 2 & 233. & 4 & 1 & 3 & 4 & 4 & 4 & 3 & 1 & 2 & 1 & 4 & 1 \\
\hline 68. & 3 & 1 & 3 & 2 & 2 & 2 & 4 & 1 & 4 & 1 & 4 & 1 & 234. & 4 & 1 & 4 & 4 & 4 & 3 & 3 & 1 & 2 & 1 & 4 & 1 \\
\hline 69. & 3 & 1 & 4 & 2 & 3 & 3 & 2 & 1 & 2 & 1 & 4 & 1 & 235. & 4 & 1 & 3 & 4 & 4 & 4 & 3 & 1 & 2 & 3 & 4 & 1 \\
\hline 70. & 3 & 1 & 4 & 2 & 4 & 3 & 1 & 1 & 2 & 1 & 4 & 1 & 236. & 4 & 1 & 3 & 4 & 4 & 4 & 4 & 1 & 2 & 1 & 4 & 1 \\
\hline 71. & 3 & 1 & 4 & 2 & 4 & 2 & 3 & 1 & 3 & 1 & 4 & 1 & 237. & 4 & 1 & 4 & 4 & 4 & 2 & 4 & 1 & 3 & 2 & 4 & 1 \\
\hline 72. & 3 & 1 & 3 & 3 & 1 & 4 & 2 & 1 & 2 & 1 & 4 & 1 & 238. & 4 & 2 & 2 & 0 & 0 & 0 & 0 & 0 & 0 & 2 & 4 & 1 \\
\hline 73. & 3 & 1 & 2 & 3 & 1 & 3 & 3 & 1 & 4 & 1 & 4 & 1 & 239. & 4 & 2 & 3 & 0 & 0 & 0 & 1 & 1 & 2 & 1 & 4 & 1 \\
\hline 74. & 3 & 1 & 3 & 3 & 1 & 4 & 3 & 1 & 2 & 1 & 4 & 1 & 240 . & 4 & 2 & 3 & 0 & 0 & 0 & 2 & 1 & 1 & 2 & 4 & 2 \\
\hline 75. & 3 & 1 & 2 & 3 & 1 & 3 & 4 & 1 & 4 & 1 & 4 & 1 & 241. & 4 & 2 & 3 & 0 & 0 & 0 & 2 & 1 & 2 & 2 & 4 & 1 \\
\hline 76. & 3 & 1 & 2 & 3 & 2 & 3 & 0 & 0 & 0 & 1 & 4 & 1 & 242. & 4 & 2 & 3 & 0 & 0 & 0 & 3 & 1 & 2 & 1 & 4 & 1 \\
\hline 77. & 3 & 1 & 3 & 3 & 2 & 4 & 2 & 1 & 2 & 1 & 4 & 1 & 243. & 4 & 2 & 3 & 0 & 0 & 0 & 3 & 1 & 2 & 2 & 4 & 1 \\
\hline 78. & 3 & 1 & 4 & 3 & 2 & 3 & 2 & 1 & 2 & 1 & 4 & 1 & 244. & 4 & 2 & 3 & 0 & 0 & 0 & 4 & 1 & 2 & 1 & 4 & 1 \\
\hline 79. & 3 & 1 & 2 & 3 & 2 & 4 & 3 & 1 & 3 & 1 & 4 & 1 & 245. & 4 & 2 & 2 & 0 & 0 & 0 & 4 & 1 & 3 & 2 & 4 & 1 \\
\hline 80. & 3 & 1 & 3 & 3 & 2 & 4 & 3 & 1 & 2 & 2 & 4 & 1 & 246. & 4 & 2 & 3 & 1 & 3 & 2 & 0 & 0 & 0 & 1 & 3 & 1 \\
\hline 81. & 3 & 1 & 3 & 3 & 2 & 2 & 4 & 1 & 4 & 1 & 4 & 1 & 247. & 4 & 2 & 4 & 2 & 1 & 3 & 2 & 1 & 1 & 2 & 4 & 2 \\
\hline 82. & 3 & 1 & 2 & 3 & 3 & 3 & 0 & 0 & 0 & 1 & 4 & 1 & 248. & 4 & 2 & 4 & 2 & 3 & 2 & 4 & 1 & 3 & 1 & 4 & 1 \\
\hline 83. & 3 & 1 & 4 & 3 & 3 & 3 & 2 & 1 & 2 & 1 & 4 & 1 & 249. & 4 & 2 & 3 & 2 & 4 & 2 & 0 & 0 & 0 & 1 & 4 & 1 \\
\hline 84. & 3 & 1 & 3 & 3 & 3 & 4 & 3 & 1 & 2 & 1 & 4 & 1 & 250. & 4 & 2 & 4 & 3 & 2 & 3 & 1 & 1 & 2 & 1 & 4 & 1 \\
\hline 85. & 3 & 1 & 4 & 3 & 3 & 2 & 3 & 1 & 3 & 1 & 4 & 1 & 251. & 4 & 2 & 4 & 3 & 2 & 2 & 3 & 1 & 3 & 3 & 4 & 1 \\
\hline 86. & 3 & 1 & 3 & 3 & 3 & 2 & 4 & 1 & 4 & 1 & 4 & 1 & 252. & 4 & 2 & 3 & 3 & 2 & 2 & 4 & 1 & 4 & 1 & 4 & 1 \\
\hline 87. & 3 & 1 & 2 & 3 & 4 & 3 & 0 & 0 & 0 & 1 & 4 & 1 & 253. & 4 & 2 & 3 & 3 & 3 & 2 & 0 & 0 & 0 & 1 & 4 & 1 \\
\hline 88. & 3 & 1 & 3 & 3 & 4 & 4 & 1 & 1 & 2 & 1 & 4 & 1 & 254. & 4 & 2 & 4 & 3 & 3 & 3 & 1 & 1 & 1 & 2 & 4 & 2 \\
\hline 89. & 3 & 1 & 4 & 3 & 4 & 3 & 2 & 1 & 2 & 1 & 4 & 1 & 255. & 4 & 2 & 4 & 3 & 3 & 3 & 2 & 1 & 2 & 1 & 4 & 1 \\
\hline 90. & 3 & 1 & 2 & 3 & 4 & 4 & 3 & 1 & 3 & 1 & 4 & 1 & 256. & 4 & 2 & 4 & 3 & 3 & 3 & 3 & 1 & 2 & 1 & 4 & 1 \\
\hline 91. & 3 & 1 & 2 & 3 & 4 & 3 & 4 & 1 & 4 & 1 & 4 & 1 & 257. & 4 & 2 & 3 & 3 & 3 & 2 & 4 & 1 & 4 & 1 & 4 & 1 \\
\hline 92. & 3 & 1 & 3 & 4 & 1 & 4 & 1 & 1 & 1 & 1 & 4 & 2 & 258. & 4 & 2 & 4 & 3 & 3 & 2 & 4 & 1 & 3 & 1 & 4 & 1 \\
\hline 93. & 3 & 1 & 3 & 4 & 1 & 4 & 2 & 1 & 2 & 1 & 4 & 1 & 259. & 4 & 2 & 4 & 3 & 4 & 3 & 1 & 1 & 1 & 1 & 4 & 2 \\
\hline 94. & 3 & 1 & 2 & 4 & 1 & 3 & 4 & 1 & 4 & 1 & 4 & 1 & 260. & 4 & 2 & 4 & 3 & 4 & 3 & 1 & 1 & 1 & 2 & 4 & 2 \\
\hline 95. & 3 & 1 & 3 & 4 & 2 & 4 & 2 & 1 & 2 & 1 & 4 & 1 & 261. & 4 & 2 & 4 & 3 & 4 & 3 & 2 & 1 & 2 & 1 & 4 & 1 \\
\hline 96. & 3 & 1 & 3 & 4 & 2 & 4 & 2 & 1 & 2 & 2 & 4 & 1 & 262. & 4 & 2 & 4 & 3 & 4 & 3 & 3 & 1 & 2 & 1 & 4 & 1 \\
\hline 97. & 3 & 1 & 3 & 4 & 2 & 4 & 3 & 1 & 2 & 1 & 4 & 1 & 263. & 4 & 2 & 2 & 4 & 1 & 3 & 0 & 0 & 0 & 2 & 4 & 1 \\
\hline 98. & 3 & 1 & 3 & 4 & 2 & 4 & 3 & 1 & 2 & 2 & 4 & 1 & 264. & 4 & 2 & 3 & 4 & 1 & 4 & 1 & 1 & 1 & 1 & 4 & 2 \\
\hline 99. & 3 & 1 & 3 & 4 & 3 & 4 & 1 & 1 & 2 & 1 & 4 & 1 & 265. & 4 & 2 & 3 & 4 & 1 & 4 & 3 & 1 & 2 & 1 & 4 & 1 \\
\hline 100. & 3 & 1 & 3 & 4 & 3 & 4 & 3 & 1 & 2 & 1 & 4 & 1 & 266. & 4 & 2 & 2 & 4 & 1 & 4 & 4 & 1 & 3 & 1 & 4 & 1 \\
\hline 101. & 3 & 1 & 3 & 4 & 3 & 4 & 3 & 1 & 2 & 2 & 4 & 1 & 267. & 4 & 2 & 2 & 4 & 1 & 3 & 4 & 1 & 4 & 2 & 4 & 1 \\
\hline 102. & 3 & 1 & 2 & 4 & 3 & 4 & 4 & 1 & 3 & 2 & 4 & 1 & 268. & 4 & 2 & 3 & 4 & 1 & 4 & 4 & 1 & 2 & 2 & 4 & 1 \\
\hline 103. & 3 & 1 & 2 & 4 & 4 & 3 & 0 & 0 & 0 & 1 & 4 & 1 & 269. & 4 & 2 & 3 & 4 & 2 & 2 & 0 & 0 & 0 & 4 & 4 & 1 \\
\hline 104. & 3 & 1 & 3 & 4 & 4 & 4 & 1 & 1 & 1 & 1 & 4 & 2 & 270. & 4 & 2 & 3 & 4 & 2 & 4 & 1 & 1 & 1 & 1 & 4 & 2 \\
\hline 105. & 3 & 1 & 3 & 4 & 4 & 4 & 2 & 1 & 2 & 1 & 4 & 1 & 271. & 4 & 2 & 3 & 4 & 2 & 4 & 1 & 1 & 1 & 2 & 4 & 2 \\
\hline 106. & 3 & 1 & 3 & 4 & 4 & 4 & 2 & 1 & 2 & 2 & 4 & 1 & 272. & 4 & 2 & 4 & 4 & 2 & 3 & 2 & 1 & 1 & 4 & 4 & 2 \\
\hline 107. & 3 & 1 & 3 & 4 & 4 & 4 & 3 & 1 & 2 & 1 & 4 & 1 & 273. & 4 & 2 & 4 & 4 & 2 & 3 & 3 & 1 & 2 & 1 & 4 & 1 \\
\hline 108. & 3 & 1 & 2 & 4 & 4 & 4 & 4 & 1 & 3 & 1 & 4 & 1 & 274. & 4 & 2 & 3 & 4 & 2 & 4 & 3 & 1 & 2 & 2 & 4 & 1 \\
\hline 109. & 3 & 2 & 2 & 0 & 0 & 0 & 0 & 0 & 0 & 1 & 4 & 1 & 275. & 4 & 2 & 3 & 4 & 2 & 4 & 3 & 1 & 2 & 3 & 4 & 1 \\
\hline 110. & 3 & 2 & 3 & 0 & 0 & 0 & 1 & 1 & 1 & 1 & 4 & 2 & 276. & 4 & 2 & 2 & 4 & 2 & 3 & 4 & 1 & 4 & 1 & 4 & 1 \\
\hline 111. & 3 & 2 & 3 & 0 & 0 & 0 & 1 & 1 & 2 & 1 & 4 & 1 & 277. & 4 & 2 & 3 & 4 & 2 & 2 & 4 & 1 & 4 & 1 & 4 & 1 \\
\hline
\end{tabular}




\begin{tabular}{|c|c|c|c|c|c|c|c|c|c|c|c|c|c|c|c|c|c|c|c|c|c|c|c|c|c|}
\hline 112. & 3 & 2 & 2 & 0 & 0 & 0 & 3 & 1 & 3 & 1 & 4 & 1 & 278. & 4 & 2 & 3 & 4 & 2 & 4 & 4 & 1 & 2 & 1 & 4 & 1 \\
\hline 113. & 3 & 2 & 2 & 0 & 0 & 0 & 4 & 1 & 3 & 1 & 4 & 1 & 279. & 4 & 2 & 4 & 4 & 2 & 3 & 4 & 1 & 2 & 1 & 4 & 1 \\
\hline 114. & 3 & 2 & 4 & 1 & 2 & 2 & 1 & 1 & 3 & 1 & 4 & 1 & 280 . & 4 & 2 & 2 & 4 & 2 & 4 & 4 & 1 & 3 & 2 & 4 & 1 \\
\hline 115. & 3 & 2 & 3 & 2 & 3 & 2 & 0 & 0 & 0 & 1 & 4 & 1 & 281. & 4 & 2 & 3 & 4 & 3 & 2 & 0 & 0 & 0 & 1 & 4 & 1 \\
\hline 116. & 3 & 2 & 4 & 2 & 3 & 1 & 2 & 1 & 2 & 3 & 3 & 3 & 282 . & 4 & 2 & 3 & 4 & 3 & 2 & 0 & 0 & 0 & 2 & 4 & 1 \\
\hline 117. & 3 & 2 & 3 & 2 & 3 & 2 & 3 & 1 & 4 & 1 & 4 & 1 & 283. & 4 & 2 & 4 & 4 & 3 & 3 & 1 & 1 & 1 & 1 & 4 & 2 \\
\hline 118. & 3 & 2 & 3 & 3 & 1 & 4 & 1 & 1 & 1 & 1 & 4 & 2 & 284 . & 4 & 2 & 3 & 4 & 3 & 4 & 1 & 1 & 2 & 1 & 4 & 1 \\
\hline 119. & 3 & 2 & 2 & 3 & 1 & 3 & 3 & 1 & 4 & 1 & 4 & 1 & 285 . & 4 & 2 & 4 & 4 & 3 & 3 & 1 & 1 & 2 & 1 & 4 & 1 \\
\hline 120. & 3 & 2 & 2 & 3 & 1 & 3 & 4 & 1 & 4 & 1 & 4 & 1 & 286. & 4 & 2 & 3 & 4 & 3 & 4 & 2 & 1 & 2 & 1 & 4 & 1 \\
\hline 121. & 3 & 2 & 3 & 3 & 2 & 4 & 1 & 1 & 1 & 1 & 4 & 2 & 287. & 4 & 2 & 4 & 4 & 3 & 3 & 2 & 1 & 2 & 1 & 4 & 1 \\
\hline 122. & 3 & 2 & 4 & 3 & 2 & 3 & 2 & 1 & 2 & 1 & 4 & 1 & 288. & 4 & 2 & 3 & 4 & 3 & 4 & 2 & 1 & 1 & 2 & 4 & 2 \\
\hline 123. & 3 & 2 & 3 & 3 & 2 & 4 & 3 & 1 & 2 & 1 & 4 & 1 & 289. & 4 & 2 & 4 & 4 & 3 & 3 & 2 & 1 & 1 & 2 & 4 & 2 \\
\hline 124. & 3 & 2 & 2 & 3 & 3 & 3 & 0 & 0 & 0 & 1 & 4 & 1 & 290. & 4 & 2 & 4 & 4 & 3 & 3 & 2 & 1 & 2 & 2 & 4 & 1 \\
\hline 125. & 3 & 2 & 3 & 3 & 3 & 2 & 0 & 0 & 0 & 1 & 4 & 1 & 291. & 4 & 2 & 4 & 4 & 3 & 3 & 3 & 1 & 2 & 1 & 4 & 1 \\
\hline 126. & 3 & 2 & 4 & 3 & 3 & 3 & 1 & 1 & 1 & 1 & 4 & 2 & 292. & 4 & 2 & 3 & 4 & 3 & 4 & 3 & 1 & 2 & 2 & 4 & 1 \\
\hline 127. & 3 & 2 & 3 & 3 & 3 & 4 & 2 & 1 & 2 & 1 & 4 & 1 & 293. & 4 & 2 & 2 & 4 & 3 & 4 & 4 & 1 & 3 & 1 & 4 & 1 \\
\hline 128. & 3 & 2 & 4 & 3 & 3 & 3 & 2 & 1 & 2 & 2 & 4 & 1 & 294. & 4 & 2 & 3 & 4 & 3 & 4 & 4 & 1 & 2 & 1 & 4 & 1 \\
\hline 129. & 3 & 2 & 2 & 3 & 3 & 4 & 3 & 1 & 3 & 1 & 4 & 1 & 295. & 4 & 2 & 2 & 4 & 3 & 3 & 4 & 1 & 4 & 2 & 4 & 1 \\
\hline 130. & 3 & 2 & 3 & 3 & 3 & 4 & 3 & 1 & 2 & 1 & 4 & 1 & 296. & 4 & 2 & 2 & 4 & 3 & 4 & 4 & 1 & 3 & 2 & 4 & 1 \\
\hline 131. & 3 & 2 & 4 & 3 & 3 & 2 & 3 & 1 & 3 & 1 & 4 & 1 & 297. & 4 & 2 & 4 & 4 & 3 & 3 & 4 & 1 & 2 & 2 & 4 & 1 \\
\hline 132. & 3 & 2 & 4 & 3 & 3 & 3 & 3 & 1 & 2 & 1 & 4 & 1 & 298. & 4 & 2 & 3 & 4 & 4 & 2 & 0 & 0 & 0 & 1 & 4 & 1 \\
\hline 133. & 3 & 2 & 3 & 3 & 3 & 4 & 3 & 1 & 2 & 2 & 4 & 1 & 299. & 4 & 2 & 2 & 4 & 4 & 3 & 0 & 0 & 0 & 2 & 4 & 1 \\
\hline 134. & 3 & 2 & 2 & 3 & 3 & 3 & 4 & 1 & 4 & 1 & 4 & 1 & 300. & 4 & 2 & 3 & 4 & 4 & 2 & 0 & 0 & 0 & 2 & 4 & 1 \\
\hline 135. & 3 & 2 & 3 & 3 & 3 & 2 & 4 & 1 & 4 & 1 & 4 & 1 & 301. & 4 & 2 & 4 & 4 & 4 & 3 & 1 & 1 & 2 & 1 & 4 & 1 \\
\hline 136. & 3 & 2 & 3 & 3 & 4 & 4 & 1 & 1 & 2 & 1 & 4 & 1 & 302. & 4 & 2 & 4 & 4 & 4 & 3 & 1 & 1 & 1 & 2 & 4 & 2 \\
\hline 137. & 3 & 2 & 3 & 3 & 4 & 4 & 2 & 1 & 2 & 1 & 4 & 1 & 303. & 4 & 2 & 3 & 4 & 4 & 4 & 2 & 1 & 2 & 1 & 4 & 1 \\
\hline 138 . & 3 & 2 & 4 & 3 & 4 & 3 & 2 & 1 & 2 & 1 & 4 & 1 & 304. & 4 & 2 & 4 & 4 & 4 & 3 & 2 & 1 & 2 & 1 & 4 & 1 \\
\hline 139. & 3 & 2 & 2 & 3 & 4 & 3 & 4 & 1 & 4 & 1 & 4 & 1 & 305. & 4 & 2 & 4 & 4 & 4 & 3 & 2 & 1 & 1 & 2 & 4 & 2 \\
\hline 140. & 3 & 2 & 2 & 3 & 4 & 3 & 4 & 1 & 4 & 2 & 4 & 1 & 306. & 4 & 2 & 3 & 4 & 4 & 4 & 3 & 1 & 2 & 1 & 4 & 1 \\
\hline 141. & 3 & 2 & 2 & 4 & 1 & 4 & 3 & 1 & 3 & 1 & 4 & 1 & 307. & 4 & 2 & 2 & 4 & 4 & 4 & 4 & 1 & 3 & 1 & 4 & 1 \\
\hline 142. & 3 & 2 & 2 & 4 & 1 & 4 & 4 & 1 & 3 & 1 & 4 & 1 & 308. & 4 & 2 & 3 & 4 & 4 & 2 & 4 & 1 & 4 & 1 & 4 & 1 \\
\hline 143. & 3 & 2 & 3 & 4 & 2 & 4 & 1 & 1 & 2 & 1 & 4 & 1 & 309. & 4 & 2 & 4 & 4 & 4 & 2 & 4 & 1 & 3 & 1 & 4 & 1 \\
\hline 144. & 3 & 2 & 3 & 4 & 2 & 4 & 3 & 1 & 2 & 1 & 4 & 1 & 310. & 4 & 2 & 2 & 4 & 4 & 4 & 4 & 1 & 3 & 2 & 4 & 1 \\
\hline 145. & 3 & 2 & 3 & 4 & 2 & 4 & 3 & 1 & 2 & 2 & 4 & 1 & 311. & 4 & 2 & 3 & 4 & 4 & 2 & 4 & 1 & 4 & 2 & 4 & 1 \\
\hline 146. & 3 & 2 & 2 & 4 & 2 & 3 & 4 & 1 & 4 & 1 & 4 & 1 & 312. & 4 & 3 & 3 & 2 & 4 & 2 & 0 & 0 & 0 & 1 & 4 & 1 \\
\hline 147. & 3 & 2 & 2 & 4 & 2 & 4 & 4 & 1 & 3 & 1 & 4 & 1 & 313. & 4 & 3 & 4 & 2 & 4 & 3 & 2 & 1 & 2 & 1 & 4 & 1 \\
\hline 148. & 3 & 2 & 3 & 4 & 3 & 4 & 1 & 1 & 1 & 2 & 4 & 2 & 314. & 4 & 3 & 4 & 3 & 3 & 2 & 3 & 1 & 3 & 1 & 4 & 1 \\
\hline 149. & 3 & 2 & 2 & 4 & 3 & 4 & 3 & 1 & 3 & 1 & 4 & 1 & 315. & 4 & 3 & 4 & 3 & 3 & 3 & 3 & 1 & 2 & 1 & 4 & 1 \\
\hline 150. & 3 & 2 & 3 & 4 & 3 & 4 & 3 & 1 & 2 & 1 & 4 & 1 & 316. & 4 & 3 & 3 & 3 & 4 & 2 & 0 & 0 & 0 & 1 & 4 & 1 \\
\hline 151. & 3 & 2 & 3 & 4 & 4 & 4 & 1 & 1 & 1 & 1 & 4 & 2 & 317. & 4 & 3 & 4 & 3 & 4 & 3 & 1 & 1 & 2 & 1 & 4 & 1 \\
\hline 152. & 3 & 2 & 3 & 4 & 4 & 4 & 1 & 1 & 2 & 1 & 4 & 1 & 318. & 4 & 3 & 3 & 3 & 4 & 2 & 4 & 1 & 4 & 1 & 4 & 1 \\
\hline 153. & 3 & 3 & 3 & 2 & 3 & 2 & 4 & 1 & 4 & 1 & 4 & 1 & 319. & 4 & 3 & 4 & 3 & 4 & 2 & 4 & 1 & 3 & 1 & 4 & 1 \\
\hline 154. & 3 & 3 & 4 & 2 & 4 & 3 & 1 & 1 & 2 & 1 & 4 & 1 & 320 . & 4 & 3 & 3 & 4 & 3 & 2 & 0 & 0 & 0 & 2 & 4 & 1 \\
\hline 155. & 3 & 3 & 4 & 3 & 3 & 3 & 2 & 1 & 2 & 1 & 4 & 1 & 321. & 4 & 3 & 4 & 4 & 3 & 3 & 3 & 1 & 2 & 1 & 4 & 1 \\
\hline 156. & 3 & 3 & 3 & 3 & 3 & 2 & 3 & 1 & 4 & 1 & 4 & 1 & 322. & 4 & 3 & 4 & 4 & 3 & 2 & 4 & 1 & 3 & 2 & 4 & 1 \\
\hline 157. & 3 & 3 & 4 & 3 & 4 & 3 & 2 & 1 & 2 & 1 & 4 & 1 & 323. & 4 & 3 & 3 & 4 & 4 & 2 & 0 & 0 & 0 & 2 & 4 & 1 \\
\hline 158. & 3 & 3 & 4 & 3 & 4 & 3 & 3 & 1 & 2 & 1 & 4 & 1 & 324. & 4 & 3 & 4 & 4 & 4 & 3 & 1 & 1 & 1 & 1 & 4 & 2 \\
\hline 159. & 4 & 1 & 2 & 0 & 0 & 0 & 0 & 0 & 0 & 1 & 4 & 1 & 325. & 4 & 3 & 4 & 4 & 4 & 3 & 1 & 1 & 2 & 1 & 4 & 1 \\
\hline 160. & 4 & 1 & 2 & 0 & 0 & 0 & 0 & 0 & 0 & 2 & 4 & 1 & 326. & 4 & 3 & 4 & 4 & 4 & 3 & 2 & 1 & 2 & 1 & 4 & 1 \\
\hline
\end{tabular}




\begin{tabular}{|l|l|l|l|l|l|l|l|l|l|l|l|l|l|l|l|l|l|l|l|l|l|l|l|l|l|l|}
\hline 161. & 4 & 1 & 3 & 0 & 0 & 0 & 1 & 1 & 1 & 1 & 4 & 2 & 327. & 4 & 3 & 4 & 4 & 4 & 3 & 2 & 1 & 1 & 2 & 4 & 2 \\
\hline 162. & 4 & 1 & 3 & 0 & 0 & 0 & 1 & 1 & 2 & 1 & 4 & 1 & 328. & 4 & 3 & 4 & 4 & 4 & 3 & 2 & 1 & 2 & 2 & 4 & 1 \\
\hline 163. & 4 & 1 & 3 & 0 & 0 & 0 & 2 & 1 & 1 & 2 & 4 & 2 & 329. & 4 & 3 & 4 & 4 & 4 & 3 & 3 & 1 & 2 & 1 & 4 & 1 \\
\hline 164. & 4 & 1 & 3 & 0 & 0 & 0 & 2 & 1 & 2 & 2 & 4 & 1 & 330. & 4 & 3 & 3 & 4 & 4 & 2 & 4 & 1 & 4 & 1 & 4 & 1 \\
\hline 165. & 4 & 1 & 3 & 0 & 0 & 0 & 3 & 1 & 2 & 3 & 4 & 1 & 331. & 4 & 3 & 3 & 4 & 4 & 2 & 4 & 1 & 4 & 2 & 4 & 1 \\
\hline 166. & 4 & 1 & 2 & 0 & 0 & 0 & 4 & 1 & 3 & 1 & 4 & 1 & 332. & 4 & 3 & 4 & 4 & 4 & 3 & 4 & 1 & 2 & 3 & 4 & 1 \\
\hline
\end{tabular}

